\title{
The Increased Sensitivity of Irregular Peripheral Canal and Otolith Vestibular Afferents Optimizes their Encoding of Natural Stimuli
}

\author{
Adam D. Schneider, ${ }^{1 \star}$ Mohsen Jamali, ${ }^{2 \star}$ Jerome Carriot, ${ }^{2}{ }^{\odot}$ Maurice J. Chacron, ${ }^{1,2}$ and $\odot$ Kathleen E. Cullen ${ }^{2}$ \\ ${ }^{1}$ Department Physics and ${ }^{2}$ Department Physiology, McGill University, Montreal, Quebec, H3A 0G4, Canada
}

\begin{abstract}
Efficient processing of incoming sensory input is essential for an organism's survival. A growing body of evidence suggests that sensory systems have developed coding strategies that are constrained by the statistics of the natural environment. Consequently, it is necessary to first characterize neural responses to natural stimuli to uncover the coding strategies used by a given sensory system. Here we report for the first time the statistics of vestibular rotational and translational stimuli experienced by rhesus monkeys during natural (e.g., walking, grooming) behaviors. We find that these stimuli can reach intensities as high as $1500 \mathrm{deg} / \mathrm{s}$ and $8 \mathrm{G}$. Recordings from afferents during naturalistic rotational and linear motion further revealed strongly nonlinear responses in the form of rectification and saturation, which could not be accurately predicted by traditional linear models of vestibular processing. Accordingly, we used linear-nonlinear cascade models and found that these could accurately predict responses to naturalistic stimuli. Finally, we tested whether the statistics of natural vestibular signals constrain the neural coding strategies used by peripheral afferents. We found that both irregular otolith and semicircular canal afferents, because of their higher sensitivities, were more optimized for processing natural vestibular stimuli as compared with their regular counterparts. Our results therefore provide the first evidence supporting the hypothesis that the neural coding strategies used by the vestibular system are matched to the statistics of natural stimuli.
\end{abstract}

Key words: natural stimuli; optimal coding; sensitivity; vestibular afferent

\section{Introduction}

Efficient processing of sensory input is critical for an organism's survival. Because some stimuli are more likely to be encountered than others, it is commonly assumed that the statistics of the natural sensory environment influence the brain's coding strategies (Attneave, 1954; Laughlin, 1981; Barlow, 2001; Simoncelli and Olshausen, 2001). However, this hypothesis remains untested for the vestibular system, which processes head motion information and thus plays a vital role in the stabilization of gaze and control of balance and posture, as well as perception of spatial orientation and self-motion. Notably, addressing whether the coding strategies used by the vestibular system are optimized to process natural stimuli first requires knowledge of the head motion experienced during natural behaviors.

The vestibular system is phylogenetically the oldest part of the inner ear and is highly conserved throughout evolution (Wallman et al., 1982). Vestibular end organ sensors detect head mo-

Received Sept. 15, 2014; revised Feb. 16, 2015; accepted Feb. 22, 2015.

Author contributions: A.D.S., M.J., J.C., M.J.C., and K.E.C. designed research; A.D.S., M.J., and J.C. performed research; A.D.S. and M.J. analyzed data; M.J.C., and K.E.C. wrote the paper.

This study was supported by Canadian Institutes of Health Research, National Institutes of Health, and FRQNT. We thank S. Nuara and W. Kucharski for excellent technical assistance.

*A.D.S. and M.J. contributed equally to this work.

The authors declare no competing financial interests.

Correspondence should be addressed to Dr. Kathleen E. Cullen, McIntyre Medical Research Building, Room 1219, 3655 Promenade Sir William 0sler, Montreal, PQ, H3G 1Y6, Canada. E-mail: kathleen.cullen@mcgill.ca.

DOI:10.1523/JNEUROSCI.3841-14.2015

Copyright $\odot 2015$ the authors $\quad 0270-6474 / 15 / 355522-15 \$ 15.00 / 0$ tion across six dimensions (three axes of translation detected by the otoliths and three axes of rotation detected by the semicircular canals; Angelaki and Cullen, 2008; Cullen, 2011). In the absence of motion, afferents display a wide range of resting discharge variability and are characterized as regular or irregular-a classification that correlates with differences in morphological features and response dynamics (Baird et al., 1988; Fernández et al., 1988; Goldberg, 2000; Straka et al., 2005). Afferent response dynamics have been traditionally measured using artificial self-motion stimuli such as sinusoids or noise with low intensity to ensure that neurons are constrained to their linear ranges (Goldberg, 2000; Sadeghi et al., 2007b; Jamali et al., 2013). To date only a few studies have reported nonlinear responses to high-intensity vestibular stimuli (Fernández and Goldberg, 1976; Sadeghi et al., 2007a). However, a recent study performed in humans has revealed that vestibular stimuli can reach large intensities during everyday activities that could elicit nonlinear responses from afferents (Carriot et al., 2014).

Thus, it currently remains unknown whether peripheral vestibular neural responses to natural input can be determined from those to artificial stimuli and whether coding strategies are constrained by the statistics of the natural environment. Here we characterized for the first time the structure of self-motion signals that are experienced by rhesus monkeys during typical natural behaviors. Existing linear models of peripheral processing incorrectly predicted that such stimuli elicit neural responses outside of the physiological range. Accordingly, we then recorded from 
afferents and developed new models incorporating static nonlinearities that accurately described responses to naturalistic stimuli. Finally, using these models, we tested whether coding by peripheral afferents is constrained by natural stimulus statistics. We found that irregular afferents, because of their higher sensitivities, were more optimized to process natural stimuli than their regular counterparts. Our results therefore have important implications for understanding the contributions of different classes of peripheral sensory neurons to the encoding of natural stimuli, and further provide evidence supporting the hypothesis that the neural coding strategies used by the vestibular system are matched to the statistics of natural stimuli.

\section{Materials and Methods}

Experimental preparation. All experimental protocols were approved by the McGill University Animal Care Committee and were in compliance with the guidelines of the Canadian Council on Animal Care. Two male macaque monkeys (Macaca fascicularis) were prepared for chronic extracellular recording using aseptic surgical techniques. The surgical preparation was similar to that previously described (Dale and Cullen, 2013). Briefly, using aseptic surgical techniques and under isoflurane anesthesia $(0.8-1.5 \%)$, a stainless steel post was secured to the animal's skull with stainless steel screws and dental acrylic resin, allowing complete immobilization of the head during the experiments. The implant also held in place a recording chamber oriented stereotaxically toward the vestibular nerve where it emerges from the internal auditory meatus. Finally, an 18-19 mm diameter eye coil (three loops of Teflon-coated stainless steel wire) was implanted in the right eye behind the conjunctiva. After the surgery, buprenorphine $(0.01 \mathrm{mg} / \mathrm{kg}, \mathrm{i} . \mathrm{m}$.) was administered as postoperative analgesia every $12 \mathrm{~h}$ for $2-5 \mathrm{~d}$ depending on the animal's pain level, and Anafen $(2 \mathrm{mg} / \mathrm{kg}$, then $1 \mathrm{mg} / \mathrm{kg}$ subsequent days) was used as an anti-inflammatory. In addition, cefazolin $(25 \mathrm{mg} / \mathrm{kg}$, i.m.) was injected twice daily for $10 \mathrm{~d}$. Animals were given at least 2 weeks to recuperate from the surgery before any experiments began.

Head movement recording. Head movements were recorded using a microelectromechanical systems (MEMS) module (iNEMO platform, STEVAL-MKI062V2; STMicroelectronics), as done recently for humans (Carriot et al., 2014). The module combined three linear accelerometers (recording linear accelerations along the fore-aft, lateral, and vertical axes) and three gyroscopes (recording angular velocity about pitch, roll, and yaw). To extend the velocity range to $\pm 2000 \mathrm{deg} / \mathrm{s}$, the MEMS module was augmented with a STEVAL-MKI107V2 three-axis gyroscope. The MEMS module, a battery, and an microSD card were encased in an extremely light $(64 \mathrm{~g})$ and small $(35 \times 35 \times 15 \mathrm{~mm})$ enclosure, which was firmly secured to the head posts of two macaque monkeys. Specifically, the plane spanned by the fore-aft and lateral axes of the MEMS module was set parallel to the horizontal stereotaxic plane (i.e., the plane passing through the inferior margin of the orbit to the external auditory meatus; Carriot et al., 2014). Data from each of the six sensors were sampled at $100 \mathrm{~Hz}$ and recorded wirelessly on an microSD card. Since we were interested in afferent responses to linear motion, we recorded the total gravito-inertial acceleration (GIA; i.e., the sum of gravity and linear motion), which is henceforth referred to as linear acceleration. Note that the data were reported in sensor coordinates in Figures 1 and 2.

Each monkey was released separately into a large familiar play cage $\left(9.5 \mathrm{~m}^{3}\right.$, with a multilevel wooden platform) where it was able to freely move and interact with another monkey from our colony for 160 min while a camera was recording its behavior. We segregated activities into three groups: (1) behaviors associated with low levels of activity that included monkey sitting observing its environment, holding and playing with objects, or grooming; (2) behaviors associated with medium levels of activity that included walking around and foraging; and (3) behaviors associated with high levels of activity that included running, jumping, and climbing, as well as rapid head shaking.
Single-unit recording. During the experiment, the head-restrained monkey was seated in a primate chair mounted on top of a vestibular table in a dimly lit room. The vestibular nerve was approached through the floccular lobe of the cerebellum, as identified by its eye-movementrelated activity (Lisberger and Pavelko, 1986; Sadeghi et al., 2007b; Jamali et al., 2013); entry to the nerve was preceded by a silence, indicating that the electrode had left the cerebellum. Extracellular single-unit activity of semicircular canal and otolith afferents was recorded using glass microelectrodes (24-27 M $\Omega$ ), the depth of which was controlled using a precision hydraulic microdrive (Narishige). Rotational head velocity and linear acceleration were measured using an angular velocity sensor (Watson) and a linear accelerometer (ADXL330Z; Analog Devices) sensor firmly secured to the animal's head post. Note that vestibular afferents could not be recorded during natural movements due to the technical difficulty of maintaining isolation from single eighth nerve afferents using high-impedance glass micropipettes while the animal underwent such complex and high-intensity dynamic stimuli. Therefore, we used naturalistic stimuli whose time course mimicked that of the natural stimuli. These were generated as done previously (Jamali et al., 2009; Brooks and Cullen, 2013; Carriot et al., 2013). As both naturalistic and natural stimuli tended to consist of excursions whose time course was approximately bell shaped, we quantified them using the following measures: intensity I (maximum value), the full width at half maximum (FWHM), and area under the curve (AuC), which were all computed from the absolute value of the stimulus.

Natural rotation stimuli displayed large heterogeneities as all three quantities were distributed over large intervals (I: 100-1500 deg/s, FWHM: 84-582 ms, AuC: 13-173 deg). The characteristics of our naturalistic rotation stimuli were all within these ranges $(\mathrm{I}=356 \pm 41 \mathrm{deg} / \mathrm{s}$, $\mathrm{FWHM}=277 \pm 19 \mathrm{~ms}$, and $\mathrm{AuC}=100 \pm 8 \mathrm{deg})$. Natural translation stimuli also displayed large heterogeneities (I: $0.8-8$ G, FWHM: 24-228 $\mathrm{ms}$, and AuC: $0.9-5.8 \mathrm{~m} / \mathrm{s}$ ). The characteristics of our naturalistic translational stimuli were also all within these ranges $(\mathrm{I}=0.87 \pm 0.35 \mathrm{G}$, $\mathrm{FWHM}=146 \pm 30 \mathrm{~ms}$, and $\mathrm{AuC}=1.2 \pm 0.3 \mathrm{~m} / \mathrm{s})$.

These stimuli were applied manually since the required velocities and accelerations were beyond the range of our controller. We focused our analysis on horizontal canal afferents $(N=11$ regular and $N=25$ irregular) and utricle otolith afferents $(N=26$ regular and $N=27$ irregular), since our motion platform was designed to apply stimuli along these axes of motion. For each afferent, stimuli were applied along the preferred axis of rotation (i.e., yaw) or translation (horizontal) as previously described (Sadeghi et al., 2007b; Jamali et al., 2013). For otolith afferents, this was achieved by rotating the monkey's head such that the neuron's preferred direction was aligned with the apparatus' axis of translation. During experimental sessions, unit activity, horizontal and vertical eye positions, and head-velocity signals were recorded on digital audiotape for later playback. During playback, action potentials from extracellular recordings were discriminated using a windowing circuit (BAK Electronics). Eye position and head-velocity signals were low-pass filtered at $250 \mathrm{~Hz}$ (eight-pole Bessel filter) and sampled at $1 \mathrm{kHz}$.

We generated binary spike trains, with $1 \mathrm{kHz}$ sampling rate for each afferent. Periods of spontaneous activity (i.e., no head movement) were used to classify afferents as regular or irregular according to the variability in their baseline spiking activity as quantified by the coefficient of variation, $\mathrm{CV}=\sigma_{I S I} / \mu_{I S I}$, where $\mu_{I S I}$ is the mean interspike interval (ISI) and $\sigma_{I S I}$ is the SD of the ISIs. Because CV varies with mean, the mean ISI, a normalized coefficient of variation $\left(\mathrm{CV}^{*}\right)$, was computed using the ISI distribution to quantify resting discharge variability as described previously (Goldberg et al., 1984). Afferents with $C V^{*}<0.1$ were classified as regular, while those with $C V^{*} \geq 0.1$ were classified as irregular as done previously (Sadeghi et al., 2007b; Jamali et al., 2013). The firing rate $r(t)$ was obtained by convolving the binary spike train with a Gaussian centered on the spike time with $10 \mathrm{~ms}$ SD. For the purposes of illustration only, we computed the cross-correlation function between the firing rate $r(t)$ and the stimulus $s(t)$ and noted the lag at which it was maximal. This lag was then used to align the response with the stimulus.

Statistical analysis of natural head movements. To test whether natural head movement signals were characterized by probability distributions that differed significantly from Normal, surrogate datasets of the same 
length with the same mean and variance but drawn from a Gaussian distribution were generated. Probability distributions for both the data and the surrogate datasets were generated with bin widths of $20 \mathrm{deg} / \mathrm{s}$ for angular velocities and $0.1 \mathrm{G}$ for linear accelerations. The deviation from normality was quantified using the kurtosis $k=\frac{\left\langle(x(t)-\mu)^{4}\right\rangle}{\sigma^{4}}$, where $x(t)$ is the signal, $\mu$ is the mean, and $\sigma$ is the SD. We note that $k=3$ for a Gaussian distribution. The signals were divided into 20 segments each lasting $\sim 8 \mathrm{~min}$ and the kurtosis values were obtained for each segment. The kurtosis values obtained from our dataset were then compared with kurtosis values obtained from the Gaussian surrogates.

Linear models of vestibular afferent responses. We first used previously established linear models to predict afferent responses to the experimentally recorded natural stimuli. Specifically, we assumed that the output firing rate $r(t)$ in response to stimulus $s(t)$ is given by the following: $r(t)=(H * s)(t)+r_{0}$, where the asterisk denotes a convolution with a filter $H(t)$ and $r_{0}$ is the baseline (i.e., in the absence of stimulation) firing rate. We used $r_{0}=104 \mathrm{~Hz}$ for canal afferents and 79 $\mathrm{Hz}$ for otolith afferents, which corresponds to average baseline firing rates observed experimentally (Sadeghi et al., 2007a; Jamali et al., 2013). Before filtering with these transfer functions, natural angular velocity recorded was projected onto the horizontal semicircular canal plane as done previously (Carriot et al., 2014) using an angle of $22^{\circ}$ nose down (Sadeghi et al., 2009).

For canal afferents, the Fourier transform of $H(t)$ (i.e., the transfer function) is given by the following:

$$
H_{\text {canal }}(f)=k \frac{S\left(S+1 / T_{1}\right)}{\left(S+1 / T_{c}\right)\left(S+1 / T_{2}\right)},
$$

with $S=i 2 \pi f$. For regular afferents, parameter values were as follows: $k=$ $2.83($ spikes $/ \mathrm{s}) /(\mathrm{deg} / \mathrm{s}), T_{1}=0.0175 \mathrm{~s}, T_{2}=0.0027 \mathrm{~s}$, and $T_{c}=5.7 \mathrm{~s}$. For irregular afferents, parameter values were as follows: $k=27.09$ (spikes/s)/ $(\mathrm{deg} / \mathrm{s}), T_{1}=0.03 \mathrm{~s}, T_{2}=0.0006 \mathrm{~s}$, and $T_{c}=5.7 \mathrm{~s}$. Overall, these parameter values are similar to those used previously (Hullar et al., 2005) and were chosen to best match available experimental data (Ramachandran and Lisberger, 2006; Sadeghi et al., 2007a).

For otoliths, we used the following transfer function (Angelaki and Dickman, 2000):

$$
H_{\text {otolith }}(f)=k \frac{S^{k_{1}}(1+a S)^{k_{2}}}{(1+b S)}
$$

with $k=59.0106($ spikes $/ \mathrm{s}) / \mathrm{G} ; k_{1}=0.0643, k_{2}=2.208, a=0.0138 \mathrm{~s}$, and $b=0.0255 \mathrm{~s}$ for regular afferents; and $k=112.7417$ (spikes/s) $/ \mathrm{G}, k_{1}=$ $0.3084, k_{2}=2.6834, a=0.0136 \mathrm{~s}$, and $b=0.0318 \mathrm{~s}$ for irregular afferents. As for canal afferents, parameter values were chosen to best match available experimental data (Jamali et al., 2013). These expressions were used to generate the linear predictions of firing rate responses to natural stimuli shown in Figure 3.

Linear-nonlinear cascade models. To characterize our experimentally observed nonlinear responses of vestibular afferents to naturalistic stimuli, we used linear-nonlinear (LN) cascade models (Chichilnisky, 2001; Massot et al., 2012) in which the firing rate response $r(t)$ is given by the following:

$$
r(t)=T\left((H * s)(t)+r_{0}\right)
$$

where $T$ is a nonlinear function. We note that, unlike previously used one-stage nonlinear models in which the firing rate at a given time is a nonlinear function of the current stimulus value (Fernández and Goldberg, 1976; Sadeghi et al., 2007a), LN models explicitly take into account neuronal temporal filtering properties (Chichilnisky, 2001) that are known to be prevalent in vestibular afferents (for review, see Goldberg, 2000). The LN model was fit to each individual recorded afferent and was used in Figures 4 and 5.

We first estimated the transfer function $H(f)$ using:

$$
H(f)=P_{s r}(f) / P_{s s}(f),
$$

where $P_{s r}(f)$ is the cross-spectrum between the stimulus s $(t)$ and binary spike train response, and $P_{s s}(f)$ is the power spectrum of the stimulus $s(t)$. Note that we only used low-amplitude portions of the stimulus to ensure that the afferent was constrained to its linear regime. The baseline firing rate was then added to this to form the linear prediction $r_{L}(t)$, which was then plotted as a function of the experimentally observed firing rate $r(t)$ to reveal the shape of the nonlinear function $T$ as done previously (Massot et al., 2012). We found that the experimental data could be well fit by a sigmoidal function:

$$
T_{\text {sig }}(x)=\frac{c_{3}}{2}\left[1+\operatorname{erf}\left(\frac{x-c_{2}}{\sqrt{2} c_{1}}\right)\right],
$$

in which $c_{1}, c_{2}$, and $c_{3}$, are fit parameters, and $\operatorname{erf}(\cdot)$ is the error function. Based on published results ( Fernández and Goldberg, 1976; Sadeghi et al., 2007a) the parameter $c_{3}$, which determines the maximum firing rate, was always $>270$ spikes/s. We also found that the variance of the firing rate distribution could be well fit by a combination of error functions:

$$
V(x)=c_{1}\left(\operatorname{erf}\left(\frac{\left(x-c_{2}\right)}{2 c_{3}^{2}}\right)+\operatorname{erf}\left(\frac{\left(c_{4}-x\right)}{2 c_{5}^{2}}\right)\right) .
$$

In practice, the first half of the experimental data was used to obtain the transfer function and the shape of the nonlinear function $T$. The LN model was then used to generate a prediction of the response to the second half of the stimulus that was compared with the experimental data. The model's performance was quantified using $R^{2}$. The linear model was generated by assuming that $T(x)=x$ instead and was used to generate a linear prediction of the response that was then also compared with the experimental data and its performance was also quantified using $R^{2}$. Note that the functions plotted in Figure 5 are population averages.

Optimal stimulus distribution that maximizes information. We used the $\mathrm{LN}$ model described above for which the response is given by the following:

$$
r(t)=T_{\text {sig }}\left((H * s)(t)+r_{0}\right),
$$

with $T_{\text {sig }}$ as a nonlinear sigmoidal function. Assuming a stimulus with probability distribution $p(s)$ and a response with probability distribution $p(r)$, the mutual information between stimulus and response is given as follows (Cover and Thomas, 1991; Rieke et al., 1996):

$$
\begin{aligned}
I(s, r) & =H(r)-H(r \mid s) \\
& =\iint d s d r p(r \mid s) p(s) \log _{2} \frac{p(r \mid s)}{p(r)},
\end{aligned}
$$

where $H(r)=-\int d r p(r) \log _{2} p(r)$ is the response entropy, $H(r \mid s)=-\int d s p(s) \int d r p(r \mid s) \log _{2} p(r \mid s)$ is the noise entropy, and $p(r \mid s)$ is the conditional response probability. As deterministic linear transformations preserve information, we have $I(s, r)=I\left(r_{L}, r\right)$. Thus, we will only consider the information between the linear prediction $r_{L}(t)=$ $(H * s)(t)+r_{0}$ and the response $r(t)=T_{\text {sig }}\left(r_{L}(t)\right)$.

In the noise-free case, there is a deterministic relationship between the linear prediction and the response and we have $p\left(r \mid r_{L}\right)=T_{\text {sig }}\left(r_{L}\right) \delta\left(r-r_{L}\right)$. The mutual information is then given by the following: $I\left(r_{L}, r\right)=H(r)$ and is maximum when (Laughlin, 1981; Wark et al., 2007):

$$
p\left(r_{L}\right)=p_{\text {opt }}\left(r_{L}\right)=T_{\text {sig }}^{\prime}\left(r_{L}\right) / \int_{-\infty}^{+\infty} d z T_{\text {sig }}^{\prime}(z),
$$

where $T_{\text {sig }}^{\prime}$ is the derivative of $T_{\text {sig. }}$.

If we consider that there is noise, it is not possible to find an analytical expression for the probability distribution $p_{\text {opt }}\left(r_{L}\right)$ for which the mutual information is maximum. However, previous studies have found an approximate solution if we assume that $r=T_{\text {sig }}\left(r_{L}\right)+\sqrt{V\left(r_{L}\right)} \xi$, where $\xi$ is a Normally distributed random variable with zero mean and variance unity. If the variance $V\left(r_{L}\right)$ is sufficiently small, then the optimal distribution is given by the following (Brunel and Nadal, 1998; McDonnell and Stocks, 2008): 
A

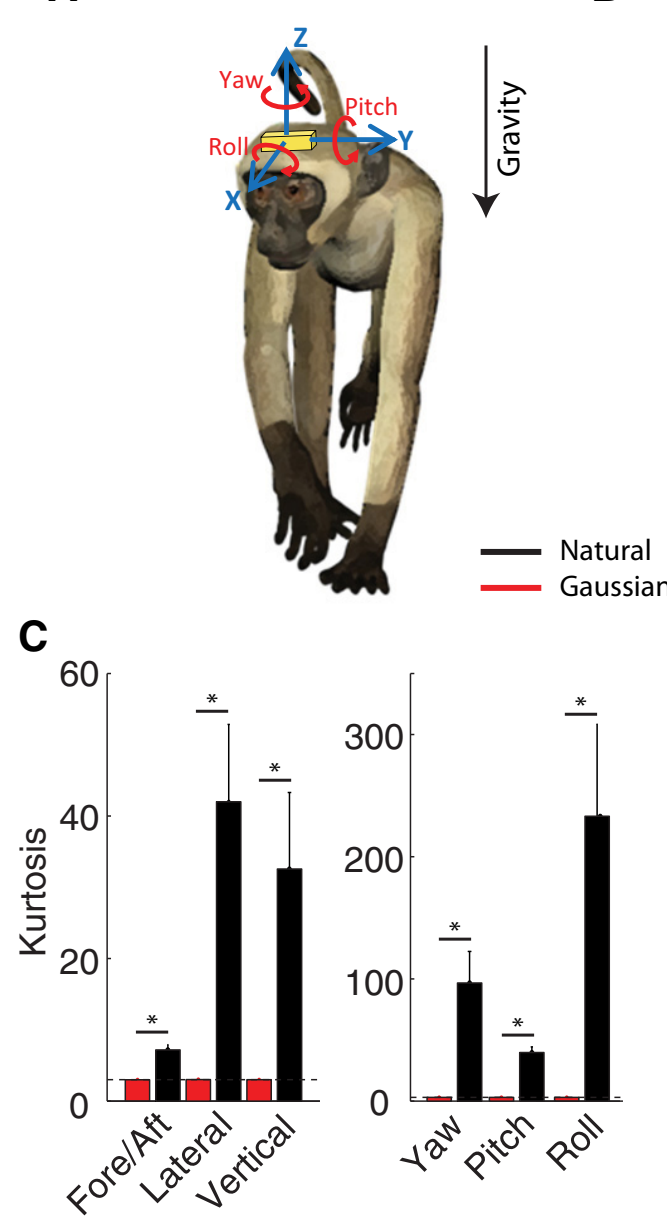

B
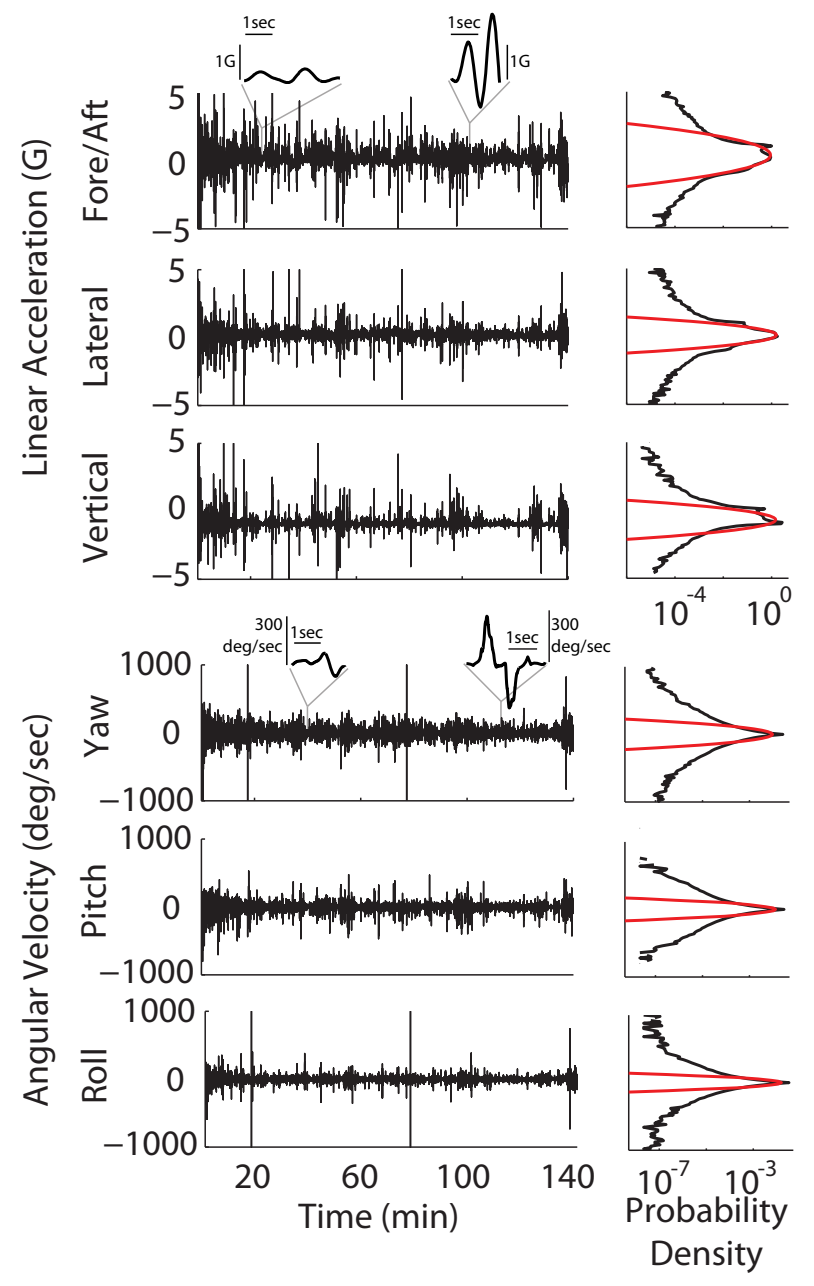

Figure 1. Statistics of natural vestibular stimuli in nonhuman primates. $A$, A MEMS module (gold box) was mounted to the monkey's head. This module recorded linear acceleration along three axes (fore/aft, lateral, and vertical) as well as angular velocity about these three axes (yaw, pitch, and roll). $\boldsymbol{B}$, Example time series for linear acceleration (top) and angular velocity (bottom) during natural motion. Natural signals displayed probability distributions (black lines) that decayed more slowly than Normal distributions (red lines). Insets show time-expanded example low-amplitude (left) and high-amplitude (right) waveforms. C, Mean kurtosis values for natural stimuli (black) and surrogate Gaussian datasets (red). Note that a Gaussian distribution has a kurtosis equal to 3 by definition. ${ }^{*} p=0.01$ level.

$$
p_{o p t}\left(r_{L}\right)=\frac{T_{s i g}^{\prime}\left(r_{L}\right)}{\sqrt{V\left(r_{L}\right)}} / \int_{-\infty}^{\infty} d z \frac{T_{s i g}^{\prime}(z)}{\sqrt{V(z)}},
$$

In comparing the expressions obtained with and without noise, it is interesting to note that the noise only appears as a normalization factor. Thus, if the noise variance is constant, then any noise intensity will not alter the shape of the optimal probability distribution.

Finally, to compute the optimal stimulus distribution $p_{\text {opt }}(s)$, we inverse filtered a signal with probability distribution $p_{\text {opt }}\left(r_{L}\right)$ whose power spectrum was equal to that of the filtered natural stimulus.

Quantifying the distance between probability distributions. To quantify the distance between two probability distributions $p(x)$ and $q(x)$, we used the Jensen-Shannon (JS) divergence defined by the following:

$$
D_{J S}(p \| q)=\left[D_{K L}(p \| m)+D_{K L}(q \| m)\right] / 2,
$$

where $m(x)=(p(x)+q(x)) / 2$ and $D_{K L}$ is the Kullback-Leibler divergence defined by the following:

$$
D_{K L}(p \| q)=\int_{-\infty}^{+\infty} d x p(x) \log _{2} \frac{p(x)}{q(x)}
$$

\section{Results}

Statistics of natural vestibular stimuli

We recorded the vestibular input experienced by freely moving rhesus monkeys during natural behaviors using a MEMS module that was attached to their heads (Fig. 1A; see Materials and Methods). The MEMS module consisted of three linear accelerometers measuring linear acceleration along the fore-aft, lateral, and vertical axes and three gyroscopes measuring angular velocity of rotations along these axes (i.e., pitch, roll, and yaw). We note that the linear acceleration signals that we recorded correspond to the total GIA (i.e., the sum of gravity and linear motion). We found that natural vestibular stimuli reached large intensities in all six axes of motion (Fig. 1B, left), and were described by probability densities that were not Gaussian as they displayed characteristically long tails (Fig. $1 B$, right). This was quantified by large kurtosis values that were all significantly greater than that obtained for a Gaussian distribution (Fig. 1C, compare black and red bars), as seen in other sensory modalities (Simoncelli and Olshausen, 2001).

To test whether specific behaviors influence stimulus statistics, we next grouped behaviors into three categories associated 
A

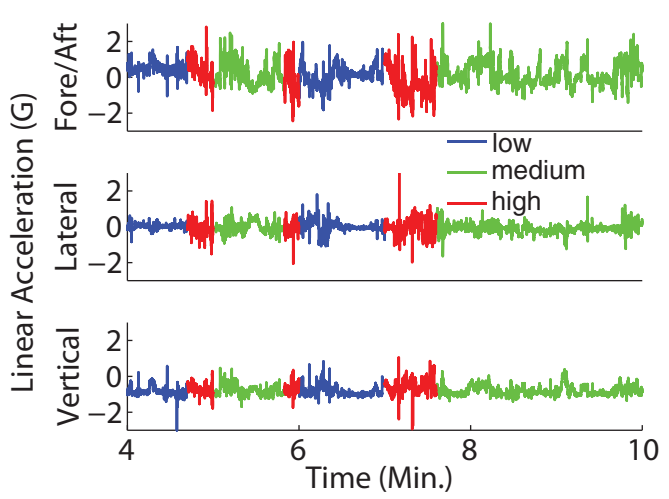

B

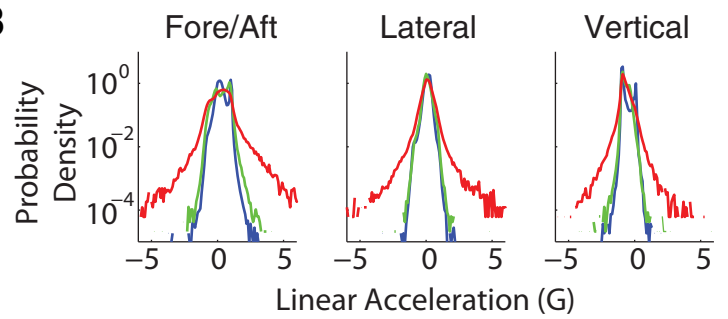

C

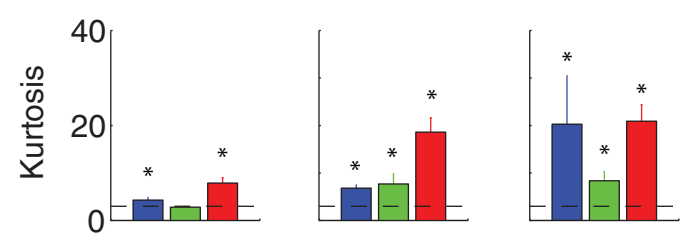

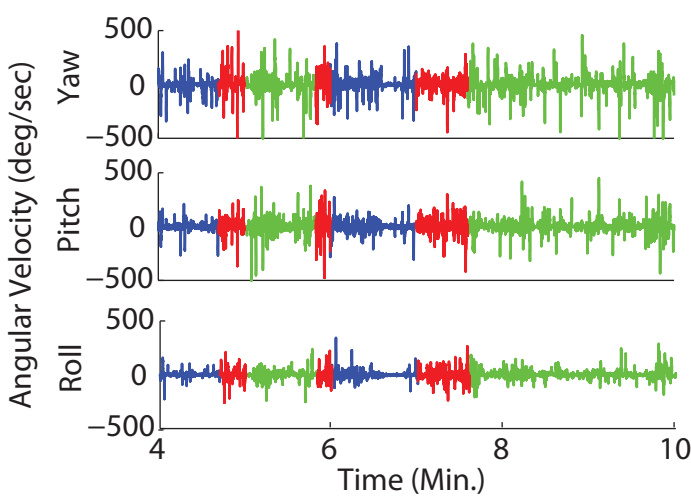
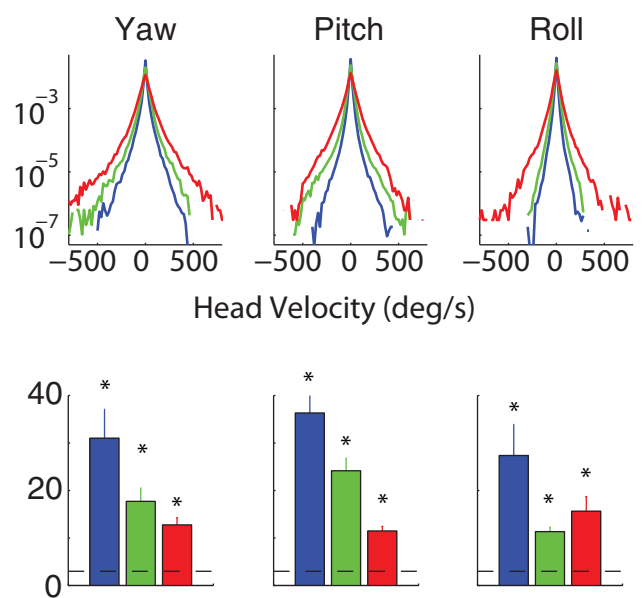

Figure 2. Natural vestibular stimulus statistics during low-, medium-, and high-activity behaviors. $A$, Example time series showing natural head acceleration and velocity traces during low (blue), medium (green), and high (red) levels of activity. B, Probability distributions of natural signals associated with low, medium, and high levels of activity. $\boldsymbol{C}$, Mean kurtosis values for natural stimuli during low (blue), medium (green), and high (red) levels of activity. The horizontal dashed line indicates the kurtosis value for a normal distribution. Asterisk indicates that the kurtosis is significantly different than that of a normal distribution using a Wilcoxon rank sum test at the $p=0.05$ level. The kurtosis was always significantly greater than that of a normal distribution except for stimuli-encountered medium levels of activity in the fore-aft translation axis.

with low, medium, and high levels of activity and quantified the statistics of the vestibular input corresponding to each category (Fig. 2A; see Materials and Methods). Specifically, periods where the monkey was sedentary and observing its environment, holding and playing with objects, or grooming behaviors were associated with low levels of activity, while those where the monkey was walking around and/or foraging were associated with medium levels of activity. Periods where the monkey was running, jumping, climbing, and was rapidly shaking its head were associated with high levels of activity. Overall, low, medium, and high levels accounted for 63,28 , and $9 \%$ of all activities, respectively. We found that the vestibular input experienced during behaviors associated with all three categories displayed probability densities that were for the most part not Gaussian (Fig. 2B) as quantified by large kurtosis values (Fig. 2C). As expected, vestibular signals associated with low, medium, and high levels of activity were distributed over progressively larger ranges across all six motion dimensions (Fig. 2B). The mean, SD, and kurtosis values obtained across low, medium, and high levels of activity for all six motion dimensions are given in Table 1.

\section{Traditional linear models of vestibular processing cannot accurately predict responses to natural stimuli}

We next tested whether previously published models of early vestibular processing that have been shown to accurately reproduce the responses of peripheral otolith and semicircular canal afferents to artificial stimuli (Goldberg, 2000; Cullen, 2004, 2011; Sadeghi et al., 2007b; Massot et al., 2011, 2012; Jamali et al., 2013) could accurately predict responses to natural stimuli. Linear systems identification techniques were used to find the optimal transfer function that best captures the input-output relationship between head motion and firing rate and predict the firing rate response, henceforth referred to as the linear prediction (Fig. $3 A$ ). We used established expressions for the transfer functions of regular and irregular semicircular canal and otolith afferents (Angelaki and Dickman, 2000; Massot et al., 2012) and fitted these to available data (Sadeghi et al., 2007a; Jamali et al., 2013). Specifically, prior studies have shown striking differences between the neural coding strategies used by semicircular canal and otolith afferents (Sadeghi et al., 2007b; Jamali et al., 2013). Most notably, while the neural sensitivities for irregular afferents are larger than those of their regular counterparts, this difference is much more pronounced for otolith than for semicircular canal afferents (Fig. 3B).

To test whether these linear models could give rise to physiologically realistic responses during natural behaviors, we used all stimulus waveforms measured during low, medium, and high levels of activity for both rotations and translations. Example stimulus waveforms used are shown in the top of Figure $3 C$ for yaw rotations (left) and fore-aft translations (right). We found negative linear firing rate predictions for both otolith and semicircular canal afferents, which is of course outside of the physio- 
Table 1. Averaged mean, SD, and kurtosis values of natural movements during low, medium, high, and all levels of activity

\begin{tabular}{lrlr}
\hline & Mean \pm SE & SD \pm SE & Kurtosis \pm SE \\
\hline Fore-aft & & & \\
All & $0.34 \pm 0.05$ & $0.44 \pm 0.02$ & $7.18 \pm 0.72$ \\
Low & $0.37 \pm 0.06$ & $0.31 \pm 0.02$ & $4.30 \pm 0.56$ \\
Medium & $0.36 \pm 0.04$ & $0.48 \pm 0.02$ & $2.81 \pm 0.20$ \\
High & $0.28 \pm 0.05$ & $0.68 \pm 0.03$ & $7.86 \pm 1.13$ \\
Lateral & & & \\
All & $0.12 \pm 0.03$ & $0.25 \pm 0.02$ & $42.00 \pm 10.84$ \\
Low & $0.12 \pm 0.03$ & $0.17 \pm 0.01$ & $6.80 \pm 0.71$ \\
Medium & $0.07 \pm 0.03$ & $0.20 \pm 0.01$ & $7.69 \pm 2.11$ \\
High & $0.11 \pm 0.03$ & $0.41 \pm 0.03$ & $18.59 \pm 3.03$ \\
Vertical & & & \\
All & $-0.71 \pm 0.05$ & $0.27 \pm 0.02$ & $32.57 \pm 10.73$ \\
Low & $-0.71 \pm 0.06$ & $0.22 \pm 0.03$ & $20.26 \pm 10.24$ \\
Medium & $-0.72 \pm 0.02$ & $0.23 \pm 0.01$ & $8.36 \pm 1.93$ \\
High & $-0.70 \pm 0.02$ & $0.36 \pm 0.02$ & $20.89 \pm 3.45$ \\
Yaw & & & \\
All & $-0.04 \pm 0.02$ & $42.07 \pm 3.77$ & $96.69 \pm 25.77$ \\
Low & $-0.13 \pm 0.07$ & $25.45 \pm 2.08$ & $31.00 \pm 6.07$ \\
Medium & $0.23 \pm 0.26$ & $43.05 \pm 2.43$ & $17.71 \pm 2.77$ \\
High & $-0.58 \pm 0.93$ & $74.08 \pm 7.87$ & $12.75 \pm 1.49$ \\
Pitch & & & \\
All & $0.05 \pm 0.01$ & $31.34 \pm 2.22$ & $39.72 \pm 4.66$ \\
Low & $0.04 \pm 0.04$ & $17.94 \pm 1.45$ & $36.31 \pm 6.82$ \\
Medium & $0.18 \pm 0.09$ & $35.40 \pm 1.91$ & $24.16 \pm 2.57$ \\
High & $-0.34 \pm 0.45$ & $65.02 \pm 3.05$ & $11.47 \pm 0.96$ \\
Roll & & & \\
All & $-0.04 \pm 0.01$ & $25.45 \pm 2.09$ & $233.11 \pm 75.32$ \\
Low & $0.05 \pm 0.06$ & $13.10 \pm 0.97$ & $27.35 \pm 6.55$ \\
Medium & $-0.00 \pm 0.19$ & $24.35 \pm 1.26$ & $11.34 \pm 0.93$ \\
High & $-0.65 \pm 0.58$ & $51.43 \pm 5.39$ & $15.64 \pm 3.06$ \\
\hline
\end{tabular}

Values are expressed in $\mathrm{G}$ for translations (i.e., fore-aft, lateral, and vertical) and deg/s for rotations (yaw, pitch, and roll).

logical range. The middle and bottom of Figure $3 C$ show the responses of regular and irregular semicircular canal (left) and otolith (right) afferents to the example waveforms, respectively. Moreover, the models' tendency to incorrectly predict negative firing rates was greater for stimuli encountered during high levels of activity (e.g., $1500 \mathrm{deg} / \mathrm{s}$ and $8 \mathrm{G}$ ). For the same stimulation, linear models also showed a greater tendency to incorrectly predict negative firing rates for irregular afferents due to their larger sensitivities. Quantification of the percentage of time during which the firing rate prediction is negative revealed low values $(<5 \%)$ across all six motion dimensions for regular afferents. However, for irregular afferents, these values exceeded $20 \%$ for high levels of activity (Fig. 3D). We conclude that existing linear models of vestibular afferent responses (for review, see Goldberg, 2000) cannot reliably predict responses to natural stimuli, and that this is especially the case for irregular afferents during the high-amplitude stimuli associated with high levels of activity.

\section{Building LN models of peripheral vestibular afferent responses to naturalistic stimuli}

It follows that if our conclusion above is correct, then natural stimuli will elicit nonlinear responses from vestibular afferents. To explicitly test this, we recorded afferent responses to highintensity naturalistic stimuli consisting of either rotation or linear motion whose time course mimicked that seen during natural movements (Fig. 1B, insets). We then used LN models (Chichilnisky, 2001) to characterize the nature of the nonlinearity in the input-output relationship of vestibular afferents (Fig. 4A). These models assume that the output firing rate is calculated by first linearly filtering the input stimulus via a transfer function and then passing the resulting linear prediction through a static nonlinear function (Fig. 4A, nonlinear prediction; see Materials and Methods). In practice, for each canal and otolith afferent (Fig. 4B), we estimated the transfer function from the response to the low-amplitude portions of the stimulus (see Materials and Methods). We then estimated the shape of the nonlinearity by plotting the actual firing rate as a function of the linear prediction from the whole dataset. Figure $4 C$ compares the performance of both linear and LN models when predicting the firing rate response of an example irregular otolith afferent to naturalistic stimuli. We found that both models provided comparable predictions for low-amplitude stimulus segments (Fig. 4C, left). In contrast, we found that the LN model gave a better prediction of the response to high-amplitude stimulus segments (Fig. 4C, right) as quantified by more than a twofold larger $R^{2}$ value (Fig. $4 D$, inset). This is because the afferent displays cutoff (i.e., ceases to fire action potentials) and saturation during the large negative and positive portions of the stimulus, respectively, which cannot be predicted from the linear model alone (Fig. 4C). Consequently, the firing rate response as a function of the linear prediction showed strong deviations from the identity line that were well fit by a sigmoidal function (Fig. 4D). Qualitatively similar results were seen for an example irregular canal afferent (Fig. $4 E, F)$. Thus, LN cascade models can accurately predict afferent responses to high-amplitude naturalistic stimuli mimicking natural signals. Figure 5 shows the results of this same analysis for our populations of canal and otolith afferents where the population-averaged actual firing rate is plotted as a function of the linear prediction. On average, the goodness of fit of LN models was always significantly larger than that of linear models for both regular (Fig. $5 A, C$ ) and irregular (Fig. $5 B, D$ ) afferents. However, this difference was most striking for irregular afferents (Fig. 5, compare $A, B$ and $C, D$ ). Accordingly, consistent with our original hypothesis, we conclude that vestibular afferents do indeed display nonlinear responses to high-amplitude naturalistic stimuli that can be accurately described by LN models.

\section{Neural heterogeneities in tuning and in trial-to-trial variability influence optimal coding}

Our above analyses of canal and otolith afferents demonstrated that those with more irregular resting discharges are likely to be driven into a nonlinear regime during naturalistic stimulation. Thus, peripheral vestibular neural responses to natural input cannot be predicted from those to low-intensity artificial stimuli. We next asked whether, and if so, how the observed differences in coding between regular and irregular afferents (i.e., likelihood of entering the nonlinear regime) as well as those seen between canal and otolith afferents (i.e., differences in sensitivity) are constrained by the statistics of natural sensory input to optimize information transmission. Specifically, are differences in resting discharge variability optimized to code for different features of natural input? Moreover, is such optimization different for canal and otolith afferents?

Theoretically, one potential optimal coding strategy is to devote the most neural resources to code for stimuli that will occur most frequently in the natural environment, which maximizes information transmission (Laughlin, 1981; Simoncelli and Olshausen, 2001; Wark et al., 2007). If we neglect trial-to-trial variability in the neural response (i.e., deterministic), then information is maximized when the firing rate response is uniformly distributed (see Materials and Methods). In this case, the stimulus distribution that maximizes information transmission (i.e., the optimal stim- 
A

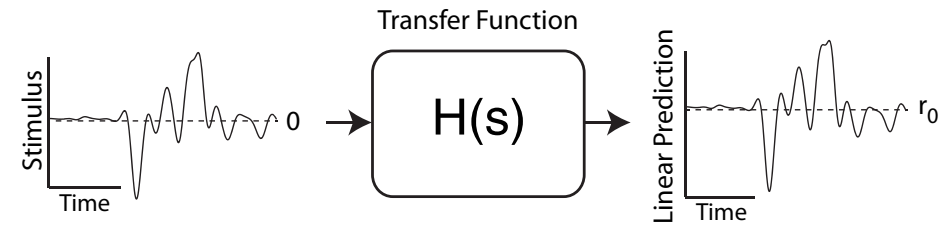

B

Canal
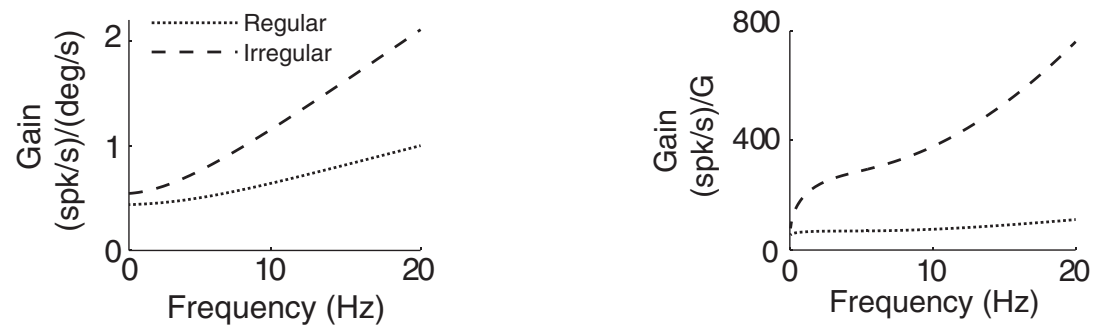

C
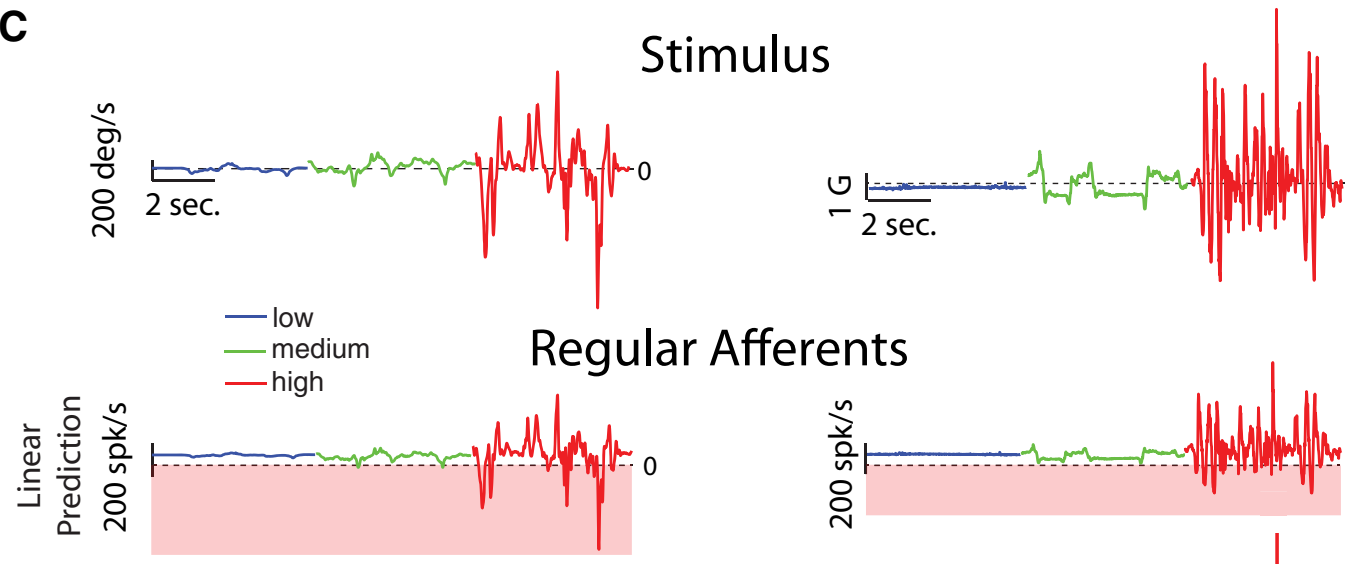

\section{Irregular Afferents}

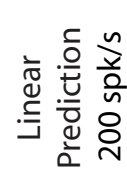

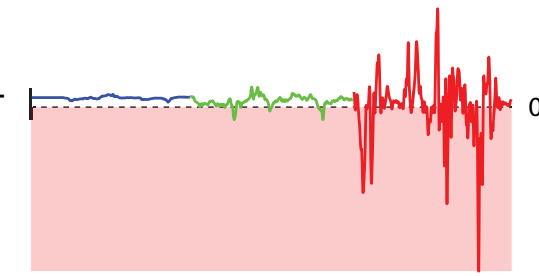

D
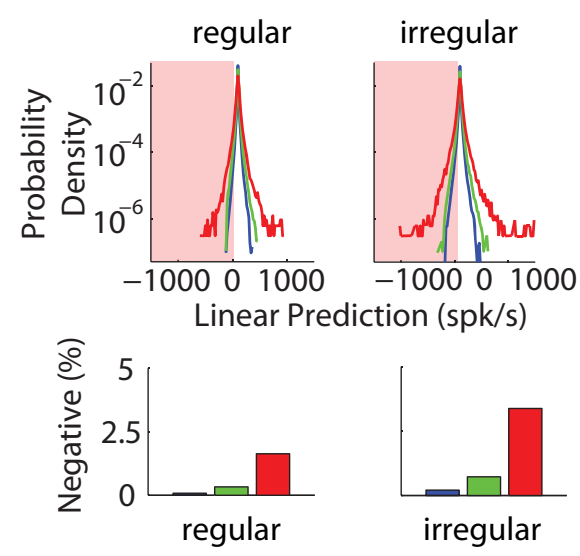
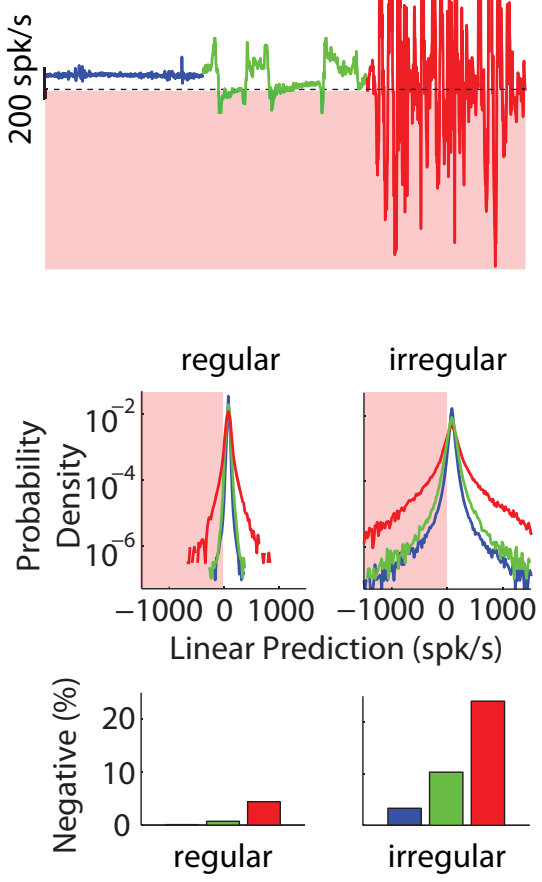
ulus distribution; see Eq. 9) is simply the derivative of the neuron's input-output relationship (Fig. $6 A$ ). Figure $6 B$ shows two different hypothetical sigmoidal input-output relationships characterized by different levels of steepness. It is thus expected that regular and irregular afferents will have different optimal stimulus distributions due to their differences in sensitivity (Fig. 3B).

Additionally, it is well known that regular and irregular afferents display different amounts of trial-to-trial variability. It is thus important to consider the influence of such variability on the optimal stimulus distribution. Theoretical studies have derived an approximate expression for the optimal stimulus distribution taking into account trial-to-trial variability in the form of variance in the firing rate response to a given stimulus (Brunel and Nadal, 1998; McDonnell and Stocks, 2008; see Materials and Methods). Figure $6 C$ shows different distributions for the firing rate variance and how they influence the optimal stimulus distribution for the input-output relationship shown in Figure 6A. In particular, if the firing rate variance is normally distributed, then the optimal stimulus distribution is wider than that obtained in the deterministic case (Fig. 6C, compare black and gray curves). In contrast, a uniform firing rate variance distribution does not alter the optimal stimulus distribution (Fig. 6C, compare black and dashed curves).

Thus, to compute the optimal stimulus distributions for different afferent classes, it is first necessary to characterize-from experimentally recorded afferent responses - the firing rate variance as a function of the stimulus to naturalistic motion. Figure $7 A$ shows the firing rate variance as a function of the stimulus for regular canal (top left), irregular canal (bottom left), regular otolith (top right), and irregular otolith (bottom right). Notably, as expected, irregular afferents displayed larger firing rate variances than regular afferents. Importantly, however, the firing rate variance decreased sharply for stimulus intensities that elicit either rectification or saturation (Fig. 5). This effect was most pronounced for irregular afferents. Figure $7 B$ shows the optimal stimulus distribution neglecting trial-to-trial variability (black curves) and taking into account the variance distributions shown in Figure 7A (dashed curves). While some differences are apparent in that the distributions taking into account trial-to-trial variability had slightly larger extent than those obtained by neglecting trial-to-trial variability, both distributions had the same shape and largely overlapped for all afferent classes. To quantify the

$\leftarrow$

Figure 3. Traditional linear models cannot accurately predict vestibular afferent responses to natural stimuli. $A$, Traditional linear models assume, to obtain the output firing rate, the stimulus is filtered by a transfer function to which a baseline value is added. $\boldsymbol{B}$, Sensitivity of regular (dotted) and irregular (dashed) afferents as a function of frequency for canal (left) and otolith (right) afferents. C, Top, Example time series showing natural stimuli associated with low (blue), medium (green), and high (red) levels of activity, for yaw rotations (left) and fore-aft translations (right). Middle, Predicted firing rate responses from the linear model for regular canal (left) and otolith (right) afferents. In all cases, the model incorrectly predicts negative firing rates (denoted by the shaded red region) during large-amplitude stimulation associated with high levels of activity. Bottom, Predicted firing rate responses from the linear model for irregular canal (left) and otolith (right) afferents. In all cases, the model incorrectly predicts negative firing rates (denoted by the shaded red region) during large-amplitude stimulation associated with high levels of activity. $\boldsymbol{D}$, Probability distributions of the linear model predictions with the shaded red region showing negative firing rates (top) and percentage of time that the firing rate signal is negative (bottom) for canal and otolith afferents for periods of low, medium, and high levels of activity (i.e., blue, green, and red curves, respectively). Data for regular and irregular afferents are shown on the left and right, respectively. Note that, in C and D, we only show data from yaw and fore-aft motion for simplicity. Similar results were observed for the other four motion dimensions. similarity between distributions, we computed the JS divergence (see Materials and Methods). We found that the JS divergence between regular and irregular afferents with variability was significantly greater than that obtained for either regular or irregular afferents with and without variability $\left(p \ll 10^{-3}\right.$ in all cases, Wilcoxon rank sum tests with Bonferroni correction; Fig. $7 B$, insets). Thus, we conclude that the different levels of trial-to-trial variability displayed by both regular and irregular canal and otolith afferents have minimal influence on the stimulus distributions that maximize information transmission.

\section{Irregular afferents are better optimized to code for natural stimuli than regular afferents}

We next explicitly tested whether and how the neural coding strategies used by the peripheral vestibular system are constrained by the statistics of stimuli encountered in the natural environment by comparing the optimal stimulus distributions obtained for each afferent class to the natural distributions. While differences in the trial-to-trial variability do not have much impact on the shape of the optimal distribution (Fig. $7 B$ ), we hypothesized that the large differences in sensitivity observed for regular and irregular afferents should strongly impact their optimal stimulus distributions. Specifically, as illustrated above in Figure $6 B$, we expected that the larger sensitivities of irregular afferents should lead to narrower optimal stimulus distributions. Figure $8 \mathrm{~A}$ shows the population-averaged optimal stimulus distributions with variability for regular (red) and irregular canal afferents (green), as well as when both populations are combined (cyan), with the natural stimulus distribution (black) superimposed. To facilitate comparison with Figure 7, these distributions are also plotted on a linear scale in the inset of Figure $8 \mathrm{~A}$. Consistent with our hypothesis, the optimal stimulus distribution for irregular afferents was indeed narrower than that obtained for regular afferents. Importantly, we further found that the optimal stimulus distribution of irregular afferents was closer to natural stimulus distribution. Overall, the JS divergence between the optimal stimulus distribution with variability and the natural distribution was always significantly lower for irregular afferents than regular afferents. This was true when we considered either the stimuli arising from all levels of activity or the stimuli arising from either low, medium, or high levels of activity alone (Fig. $8 \mathrm{~B}$ ). We performed a comparable analysis on our population of otolith afferents and obtained qualitatively similar results. Notably, the optimal stimulus distribution (with variability) of irregular otolith afferents was significantly narrower and better matched to the natural distribution in the fore-aft direction when compared with that obtained for regular afferents (Fig. $8 C$ ) as quantified by significantly lower JS values (Fig. 8D). Qualitatively similar results were obtained when comparing the optimal stimulus distributions of irregular and regular otolith afferents to the natural distribution in the lateral direction (population-averaged JS divergence values for irregular vs regular afferents; low: $0.39 \pm 0.08$ vs $0.73 \pm 0.05$, medium: $0.33 \pm 0.07$ vs $0.70 \pm 0.04$, high: $0.15 \pm$ 0.06 vs $0.56 \pm 0.07$, all: $0.30 \pm 0.07$ vs $0.67 \pm 0.05, p<0.05$ in all cases). Further, the optimal stimulus distributions obtained by pooling across all afferents (i.e., including both regular and irregular subgroups) were not better matched to the natural stimulus distribution than those obtained when only considering irregular afferents (Fig. $8 A, C$, compare green and cyan curves). Finally, we note that irregular otolith afferents were more optimized than irregular canal afferents (Fig. 8 , compare $A, C$ ) as quantified by significantly lower JS values $\left(p \ll 10^{-3}\right.$ in all cases, Wilcoxon rank sum tests). 
A

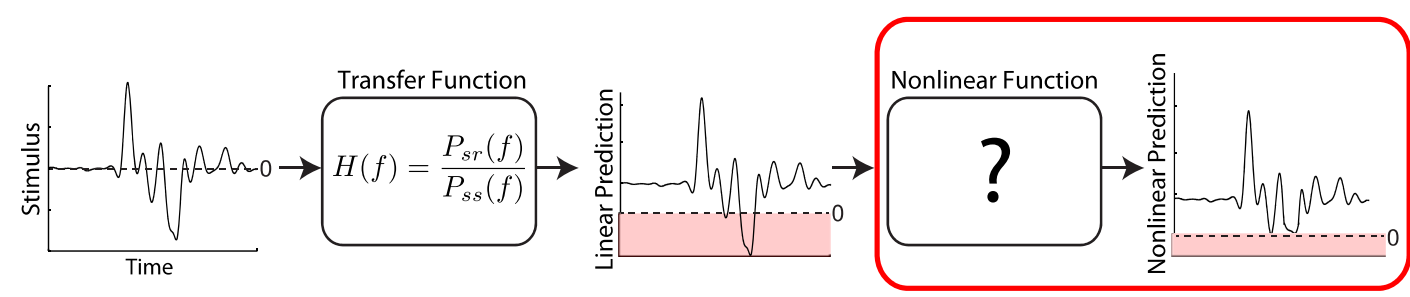

B

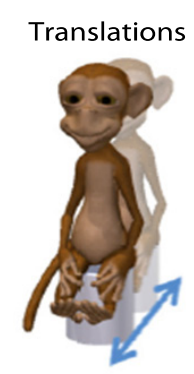

C

Low Amplitude
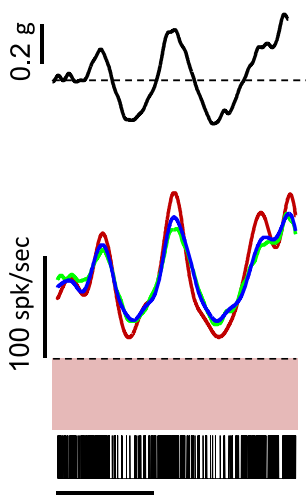

$1 \mathrm{~s}$

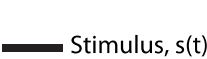

E

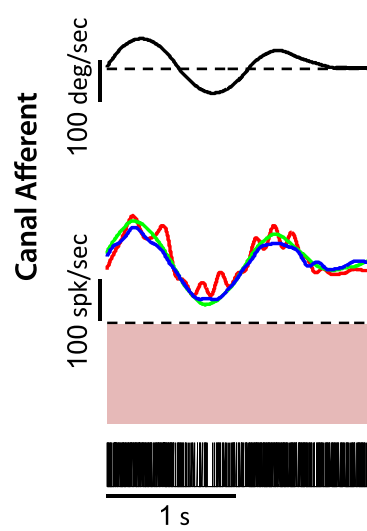

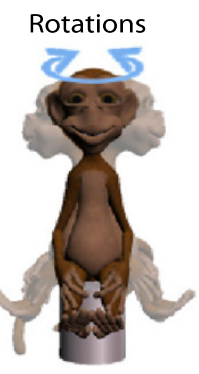

High Amplitude

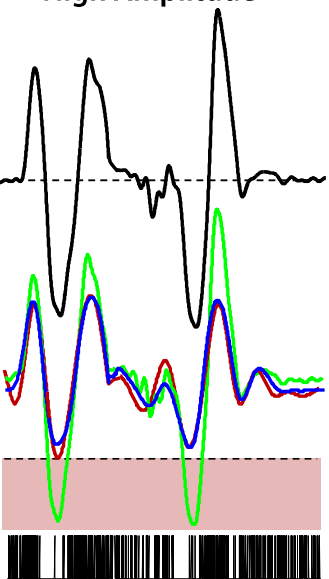

Actual Response, $r(t)$

Linear Prediction, $r_{1}(t)$

Nonlinear Prediction, $r_{N}(t)$

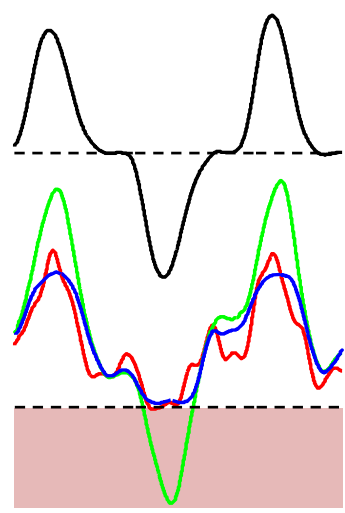

|

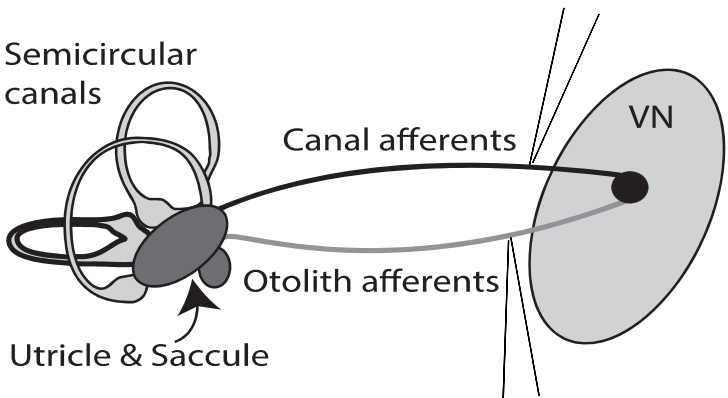

D

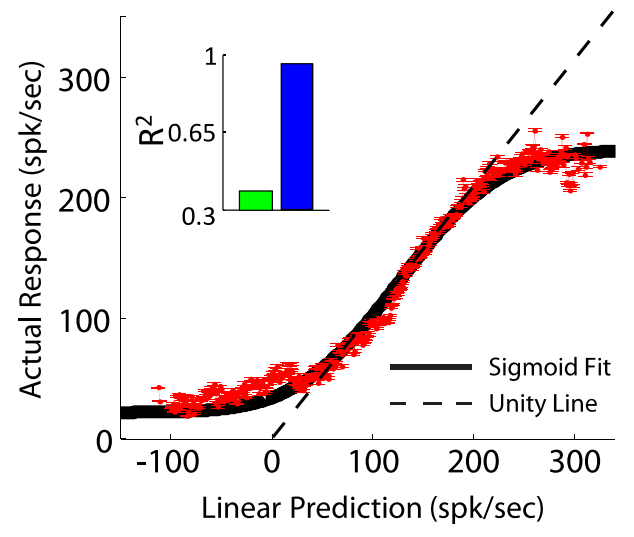

F

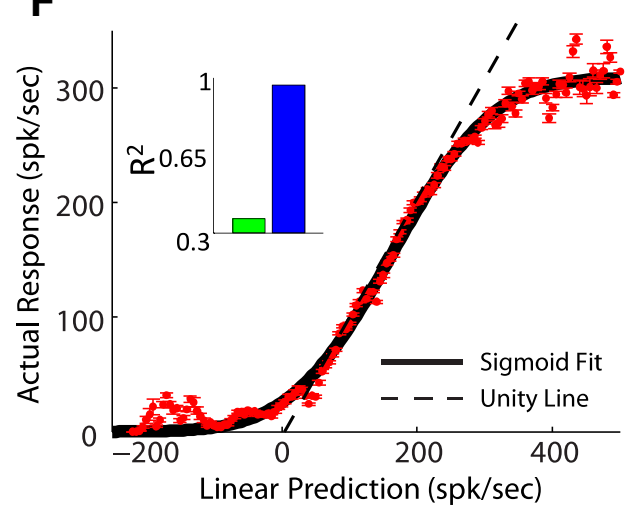

Figure 4. LN models can correctly predict vestibular afferent responses to naturalistic stimuli. $A$, Schematic showing the LN model consisting of first filtering the stimulus with a transfer function to get a linear prediction of firing rate that is then passed through a static nonlinearity to obtain the nonlinear prediction. $\boldsymbol{B}$, We recorded the responses of both otolith (Figure legend continues.) 

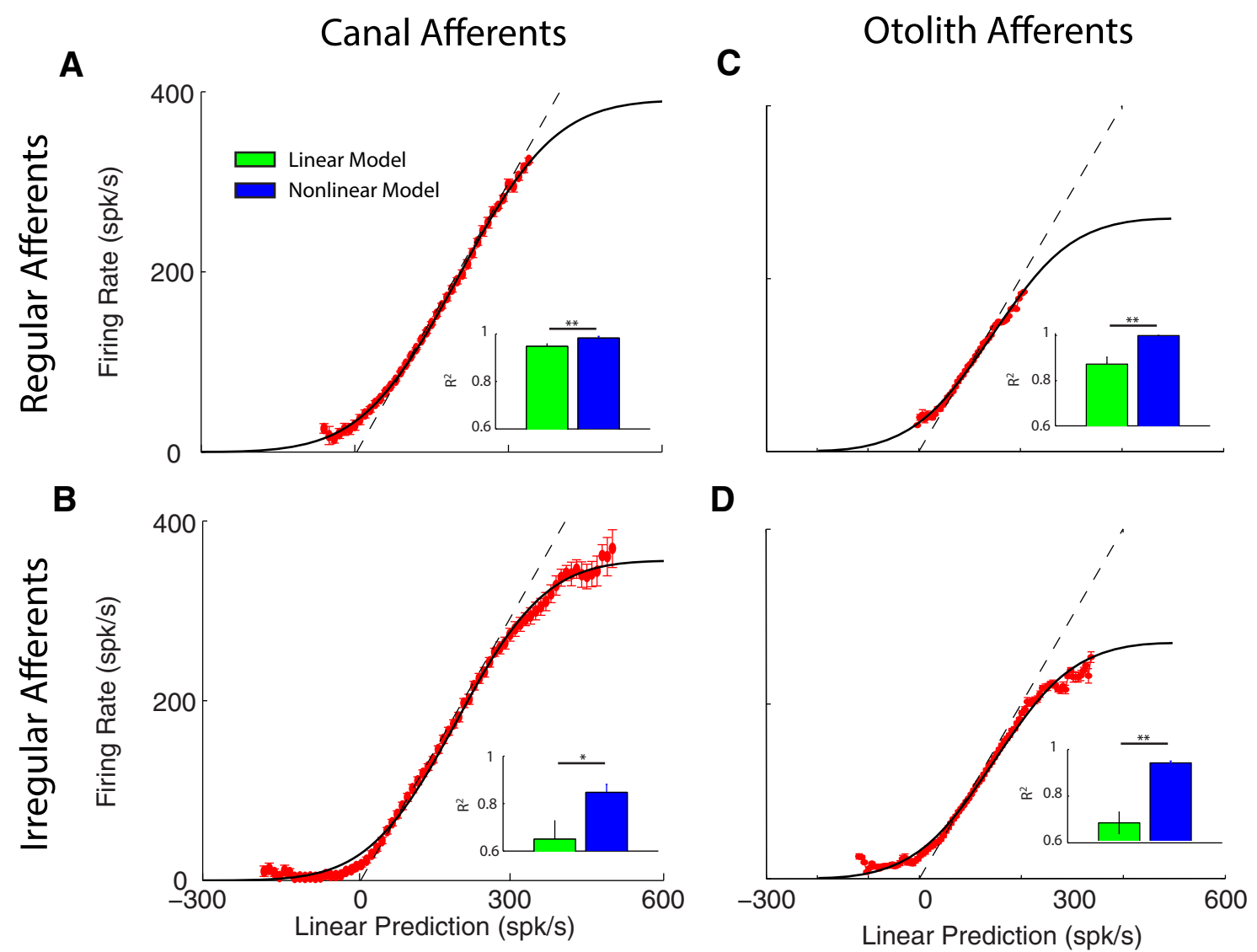

Figure 5. Regular and irregular canal and otolith afferents are well described by $L N$ models. $A-D$, Population-averaged actual response as a function of the linear prediction with best-fit sigmoid (black line) and unity line (dashed) for regular canal $(\boldsymbol{A} ; N=11)$, irregular canal $(\boldsymbol{B} ; N=25)$, regular otolith $(\boldsymbol{C} ; N=26)$, irregular otolith $(\boldsymbol{D} ; N=27)$ afferents. ${ }^{*} p=0.05$ level using a Wilcoxon rank sum test.

\section{Discussion}

\section{Summary of results}

Here we studied the statistics of natural vestibular signals experienced by monkeys to determine whether coding is optimized for such stimuli. We found that natural vestibular inputs reached large intensities as evidenced by probability distributions with long tails across all six motion dimensions, similar to those de-

$\leftarrow$

(Figure legend continued.) and canal afferent responses to naturalistic rotational and translational stimuli, respectively. VN, Vestibular nuclei. C, Low-amplitude (upper left) and highamplitude (upper right) segments of naturalistic linear acceleration stimuli (compare with insets in Fig. 1B). The bottom part shows the actual responses of an example otolith afferent (red), the linear prediction (green), and the nonlinear prediction (blue) to the low-amplitude segment (left) and to the high-amplitude segment (right). The dashed line indicates a firing rate of zero and the shaded red region indicates negative firing rates that are of course physiologically unrealistic. Also shown is the actual spiking response of the afferent (black bars). $\boldsymbol{D}$, Actual firing rate response as a function of the linear prediction for this otolith afferent reveals that the nonlinear function can be fitted by a sigmoid (black line) and deviates from the unity line (dashed). Inset, Performance as quantified by $R^{2}$ of the linear (black) and nonlinear (blue) predictions. $E$, Low-amplitude (upper left) and high-amplitude (upper right) segments of naturalistic head velocity stimuli (compare with insets in Fig. 1B). Bottom, Shows the actual responses of an example canal afferent (red), the linear prediction (green), and the nonlinear prediction (blue) to the low-amplitude segment (left) and to the high-amplitude segment (right). The dashed line indicates a firing rate of zero and the shaded red region indicates negative firing rates that are of course physiologically unrealistic. Also shown is the actual spiking response of the afferent (black bars). $\boldsymbol{F}$, Actual firing rate response as a function of the linear prediction for this canal afferent reveals that the nonlinear function can be fitted by a sigmoid (black line) and deviates from the unity line (dashed). Inset, Performance as quantified by $R^{2}$ of the linear (black) and nonlinear (blue) predictions. scribed by Carriot et al. (2014) for human. We further found that well established linear models of early vestibular processing could not predict semicircular canal or otolith afferent responses to natural vestibular stimuli. Instead they incorrectly predicted physiologically impossible negative firing rates in response to large-amplitude "off-direction" movements. This was particularly true for the coding of high-intensity activities (e.g., running, jumping, climbing, etc.) by irregular afferents. Accordingly, to develop accurate models, we recorded from afferents during naturalistic rotational and linear motion. We found that linear-nonlinear cascade models could accurately describe neural responses. Thus, we used these models to determine whether afferent coding strategies are constrained by natural stimulus statistics by computing the optimal stimulus distribution that maximizes information. We found that irregular otolith and semicircular canal afferents, due to their higher sensitivities, were better optimized to process natural stimuli. It is therefore likely that the neural coding strategies used by the vestibular system have developed to match the statistics of natural stimuli.

\section{Irregular afferents are better optimized to process natural stimuli}

There is growing evidence that sensory systems have evolved coding strategies that are adapted to optimally process natural sensory input (Laughlin, 1981; for review, see Wark et al., 2007). For example, in the fly visual system, Laughlin (1981) compared the experimentally measured neuronal input-output relationship to that which maximizes information transmission about the natural luminance distribution and found 
A
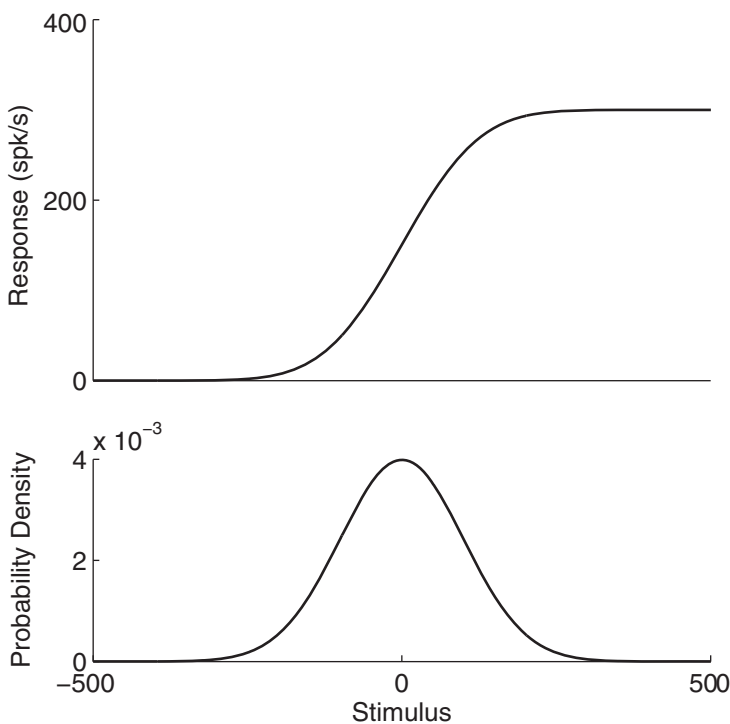

B
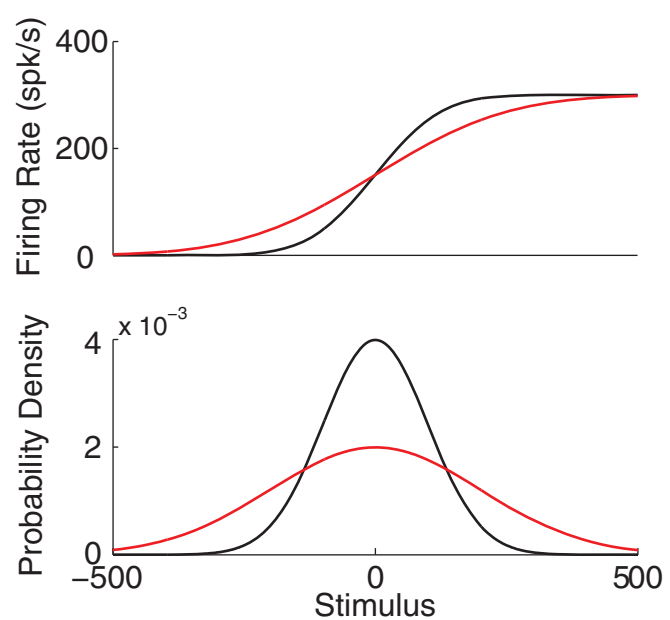

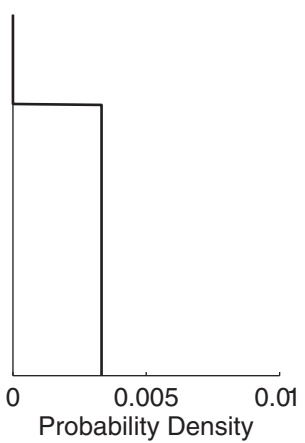

50

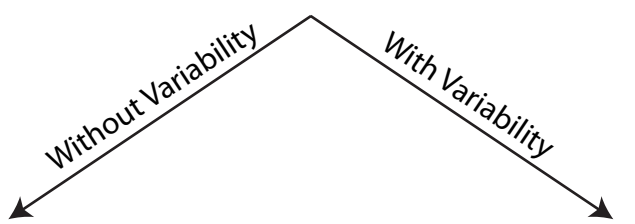

C
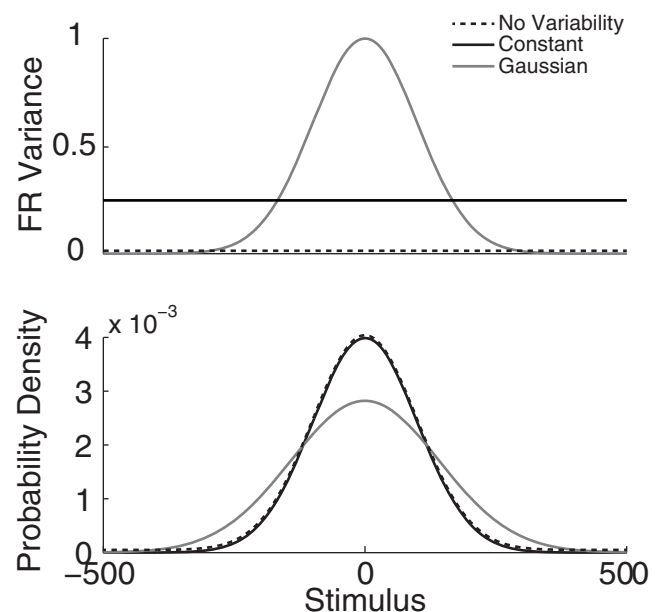

Figure 6. Hypothetical effects of neural heterogeneities in tuning and in trial-to-trial variability on optimal coding. $\boldsymbol{A}$, Differentiating the neuron's input- output relationship (top) provides the optimal stimulus distribution (bottom) that gives rise to a uniform response distribution (upper right), which maximizes information. $\boldsymbol{B}$, Input- output relationships (top) and their corresponding optimal stimulus distributions (bottom) assuming that variability is negligible. Note that the optimal stimulus distribution becomes narrower as the steepness of the input- output relationships increases (compare red and black curves). C, Input- output relationships (top) and their corresponding optimal stimulus distributions (bottom) when taking variability into account. If the firing rate variance is uniformly distributed (dashed), the optimal stimulus distribution is identical to that obtained when variability is neglected (black). In contrast, if firing rate variance is normally distributed (gray), then the optimal stimulus distribution is wider than that obtained in the deterministic case (compare gray and dashed curves).

excellent agreement between the two. Here we used a similar approach to compare the natural stimulus distribution to that which maximizes information transmission given the experimentally measured neuronal input-output relationship (Fig. 8 ) and found that irregular afferents better optimized to code for natural stimuli.

Interestingly, the evolution from stem tetrapods to amniotes was accompanied by the appearance of type I vestibular hair cells and a novel afferent terminal with a calyceal ending (for review, see Eatock and Songer, 2011). Previous studies have shown that afferents supplied by type I hair cells tend to be more irregular in their resting discharges (Goldberg and
Fernández, 1977; Si et al., 2003; Xue and Peterson, 2006). It has been hypothesized that type I hair cells evolved in amniotes as an adaptation to changes in natural stimulus statistics resulting from (1) the transition from water (i.e., characterized by resistive hydrodynamic forces) to a landbased environment (Eatock and Songer, 2011) and (2) neck elongation in amniotes (Steinacker, 2004). While it has been suggested these two factors lead to higher amplitude motion in amniotes, at least some anamniote species experience vestibular stimuli with extremely large intensities during natural self-motion (e.g., $>1000 \mathrm{deg} / \mathrm{s}$ in the spiny dogfish (Domenici et al., 2004) and $15 \mathrm{G}$ in the swimming pike (for review, see 
A

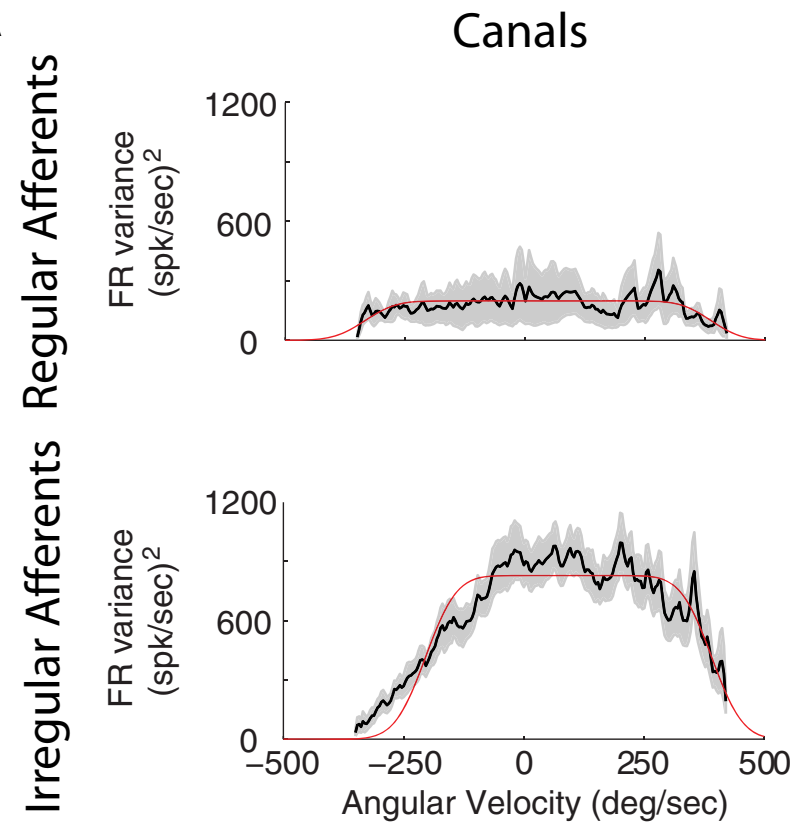

Otoliths
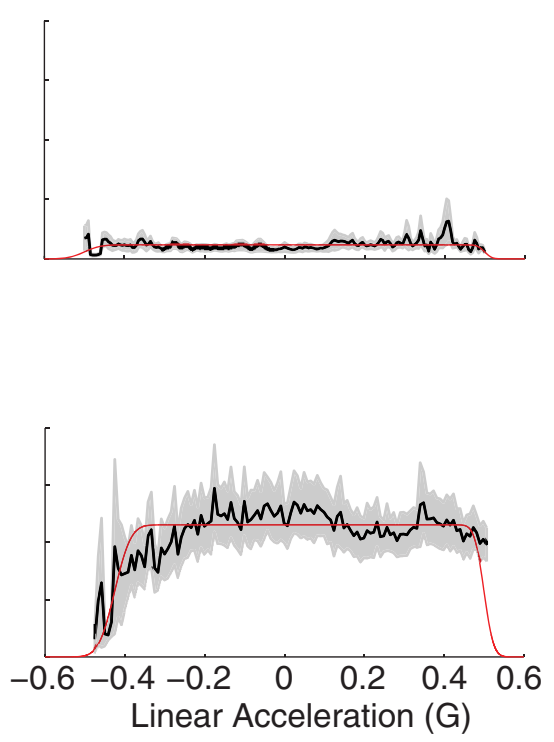

B
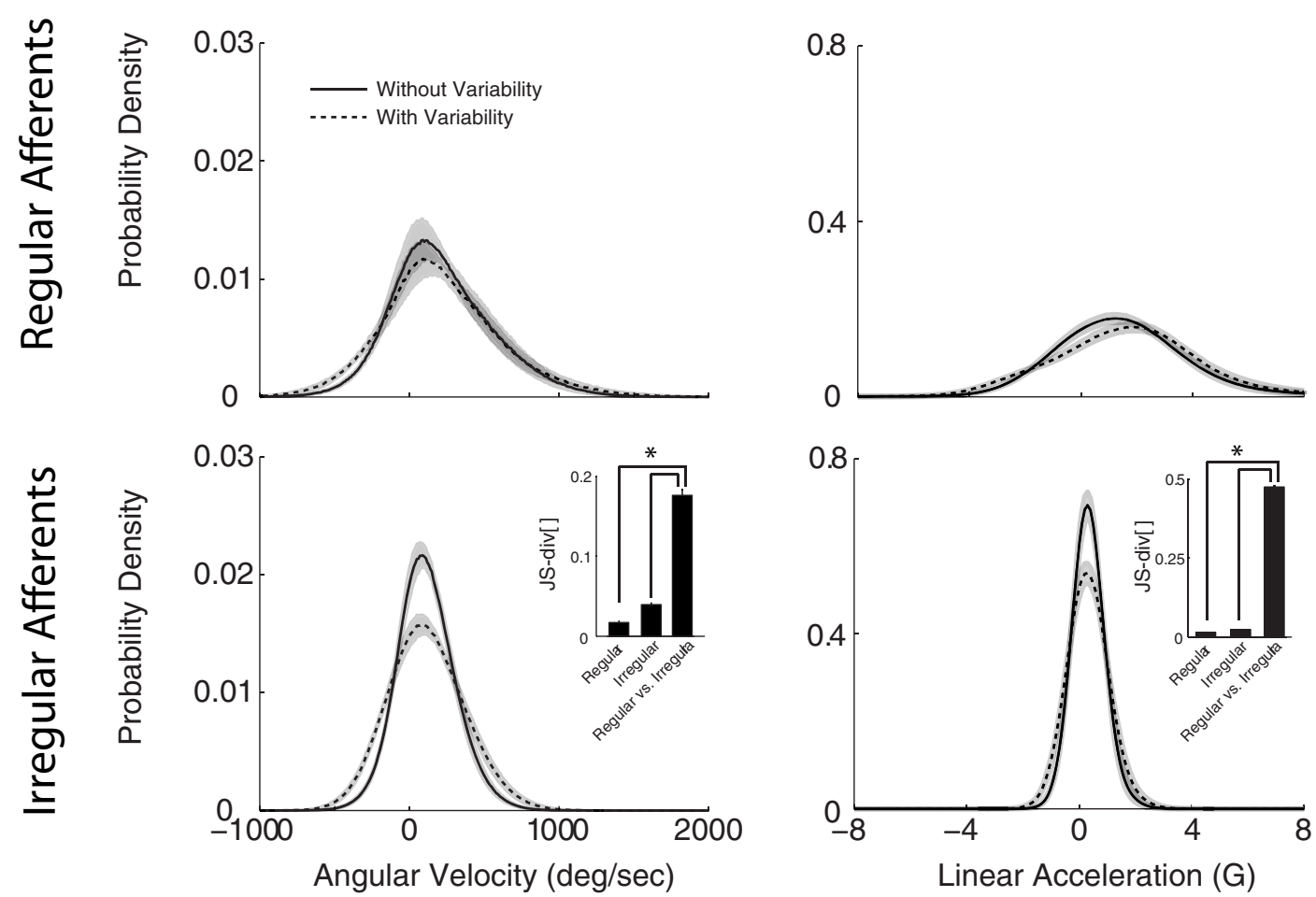

Figure 7. Effects of experimentally measured trial-to-trial variability on information transmission. $A$, Firing rate variance as a function of stimulus amplitude for canal (left) and otolith (right) afferents. Regular afferents (top) displayed less firing rate variance than irregular afferents (bottom). The red lines show the best fits (see Materials and Methods). $\boldsymbol{B}$, Including the effects of the measured firing rate variance only has minor effects on the shape of the optimal stimulus distribution as can be seen by comparing the dashed (with variability) and black (without variability) curves for regular (top) and irregular (bottom) canal (left) and otolith (right) afferents. The insets show that the population-averaged JS divergence values between regular afferents (with and without variability) and irregular afferents (with and without variability) are significantly greater than those computed between regular and irregular afferents (with variability). ${ }^{*} p=0.05$ level using a Wilcoxon rank sum test with Bonferroni correction.

Domenici and Blake, 1997). Thus, it is possible that other factors also contributed to the evolution of the vestibular periphery. For example, aquatic species might experience more constant vestibular stimulation due to water currents. Further studies comparing the statistics of natural motion across amniotes versus anamniotes are needed to address this important issue.
Nature of the neural code used by the vestibular system to transmit information about natural stimuli

It is generally thought that neurons within early vestibular pathways transmit information about head-motion stimuli in a linear fashion through their time-varying firing rates (i.e., a rate code) rather than through precise timing of action potentials (i.e., a temporal code) (Goldberg, 2000; Sadeghi et al., 

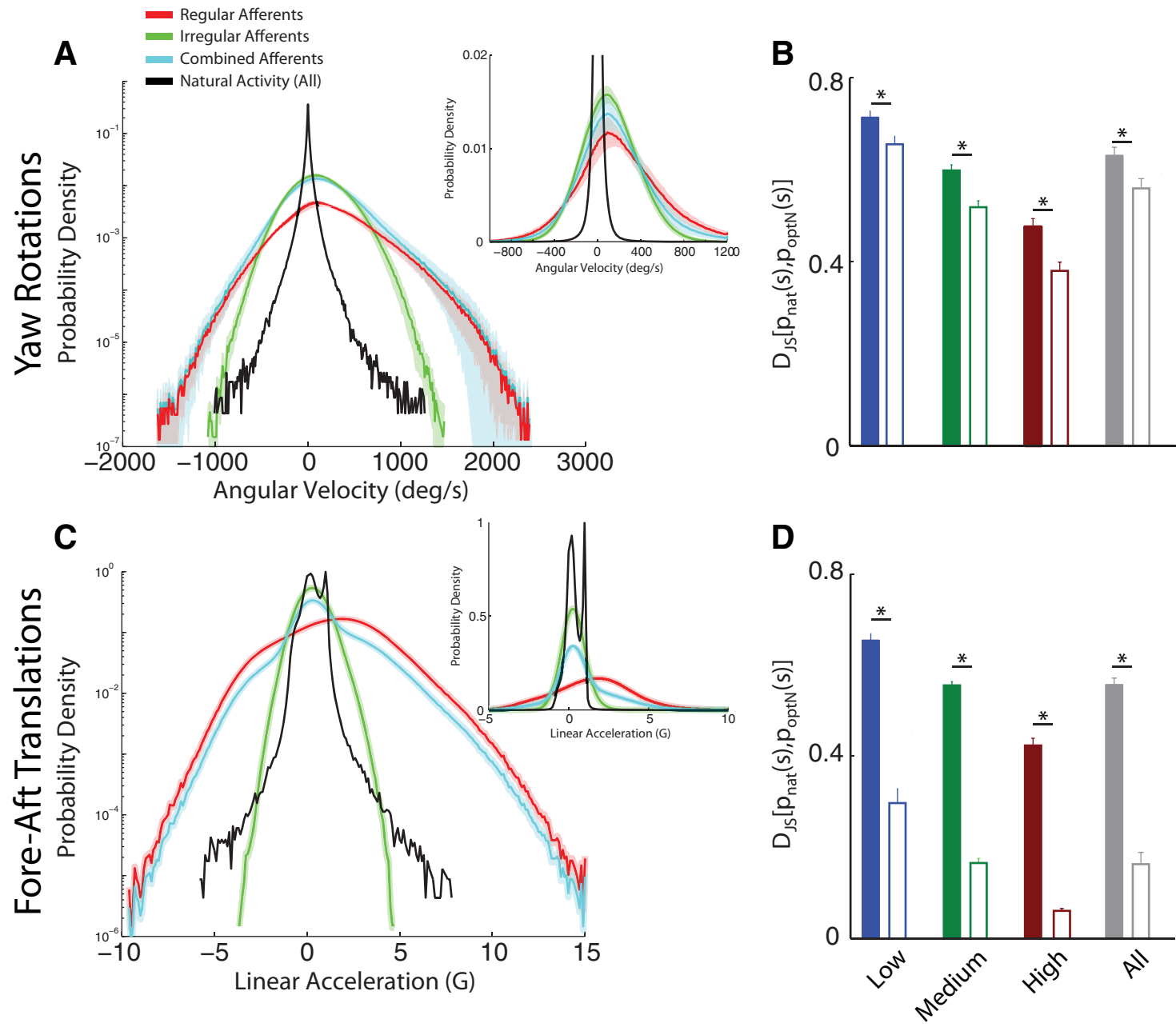

Figure 8. Irregular afferents are better optimized to code for natural stimuli than regular afferents. $\boldsymbol{A}$, Population-averaged optimal stimulus distribution for regular (red) and irregular (green), as well as for both (cyan) canal afferents. The natural stimulus distribution (solid black) is also shown. The bands show \pm 1 SEM. The inset shows the same distributions plotted on a linear scale. $\boldsymbol{B}$, Population-averaged JS divergence values quantifying the distance between the optimal stimulus distribution and the natural stimulus distribution for regular (solid bar) and irregular (empty bar) canal afferents for low (blue), medium (green), high (red), and all (black) activities. $\boldsymbol{C}, \boldsymbol{D}$, Same as $\boldsymbol{A}$ and $\boldsymbol{B}$, but for otolith afferents, respectively. ${ }^{*} p=0.05$ level using a Wilcoxon rank sum test.

2007b; Massot et al., 2011, 2012). However, previous studies have mostly focused on characterizing neuronal responses to low-amplitude artificial stimuli. As a result modulations in the firing rate were largely constrained to the neuron's linear regime and did not elicit static nonlinearities such as saturation or rectification. Interestingly, our results show that this is also often applicable to the afferent coding of natural vestibular stimuli because monkeys spend much of their time engaged in activities characterized by relatively low-amplitude head motion (i.e., sitting, grooming). However, we found that this was not true for the vestibular stimuli experienced during intermittent high-activity behaviors, such as running, because of their large amplitudes. For such behaviors, the likelihood of nonlinearities (e.g., rectification, phase locking, saturation), open the interesting possibility that the neural code used by the vestibular system to process high-amplitude stimuli is inherently different than that used for low-amplitude stimuli. Further studies will also be needed to test this interesting hypothesis.

\section{Role of variability in neural coding}

Neural variability is seen ubiquitously in the CNS but its role in neural coding is highly debated (Stein et al., 2005; Faisal et al.,
2008). The peripheral vestibular system is particularly well suited for studying the effects of variability on neural coding because regular and irregular afferents display low and high amounts of variability, respectively. While previous studies have found that variability plays an important role in vestibular coding (Goldberg, 2000; Sadeghi et al., 2007b; Massot et al., 2011, 2012; Jamali et al., 2013), our results show that sensitivity largely determines the corresponding optimal stimulus distribution (Figs. $7,8)$. Thus, from this point of view, the increased variability of irregular afferents might just be a consequence of their increased gain: the detrimental effects of such increased variability on coding and feature detection can then be reduced by averaging the activities of large afferent populations (Sadeghi et al., 2007b; Jamali et al., 2013). The fact that the increased variability and sensitivity are strongly correlated in the vestibular system (Jamali et al., 2013), as they most likely originate from intrinsic properties (Smith and Goldberg, 1986; Kalluri et al., 2010), support this hypothesis. Alternatively, it is theoretically possible that there is a source of sensory noise that is common to all afferents and that is greater for irregular afferents because of their larger sensitivity. Further studies will be needed to test these possibilities. 


\section{Coding natural stimuli by otolith versus semicircular canal afferent populations}

Previous results have shown that the coding strategies used by otolith afferents to encode linear motion differ markedly from those used by semicircular canal afferents to encode rotational motion (Sadeghi et al., 2007b; Jamali et al., 2013). Notably, strong correlations between trial-to-trial variability and sensitivity in otolith afferents are such that their ratio remains constant. As a consequence, neural detection thresholds (i.e., the minimum stimulus intensity that elicits a detectable change in neural activity) are independent of either resting discharge variability or frequency (Jamali et al., 2013). In contrast, although a strong positive correlation between variability and sensitivity is also observed for semicircular canal afferents (Goldberg, 2000), the increased sensitivity displayed by irregular semicircular canal afferents is not sufficient to compensate for their substantially higher trial-to-trial variability. As a result, irregular semicircular canal afferents display higher detection thresholds than regular ones (Sadeghi et al., 2007b; Massot et al., 2011). Interestingly, supporting these differences, we found that irregular otoliths are more optimized than irregular canal afferents, which was primarily due to the fact that the differences in sensitivity are far more pronounced in the otolith system. Nevertheless, despite these differences, we further found that both irregular semicircular canal and otolith afferents are more optimized to process natural stimuli when compared with regular afferents, thereby suggesting that both the semicircular and otolith systems have adapted to natural stimulus statistics through common principles.

\section{Implications for higher order processing of natural vestibular stimuli}

Our results have important implications for downstream processing and behavior. We have recently shown that vestibular sensory information encoded by eighth nerve afferents is nonlinearly integrated by postsynaptic neurons at the first central stage of vestibular processing (i.e., in the vestibular nuclei; Massot et al., 2012). Notably, this nonlinearity generates an intensitydependent bias in the output firing rate, when low- and highamplitude (or frequency) stimuli are presented concurrently. We speculate that this nonlinear effect will be particularly significant for high-intensity natural behaviors.

Finally, we note that although irregular afferents are better optimized to encode natural stimuli, the combined activities of both regular and irregular afferents most likely contribute to vestibular perception. Previous results obtained using artificial stimuli have led to the hypothesis that regular afferents are better suited to estimate the detailed time course of the stimulus while irregular afferents are instead better suited to detect highfrequency features (Goldberg, 2000; Sadeghi et al., 2007b; Massot et al., 2011). Such parallel processing of sensory information is a common strategy used across modalities including auditory (Takahashi et al., 1984; Oertel, 1999; Gelfand, 2004), visual (Marr, 1982; Livingstone and Hubel, 1987; Merigan and Maunsell, 1993), and electrosensory (Carr and Maler, 1986; Bell and Maler, 2005; Kawasaki, 2005), to code for different stimulus attributes. Further studies are needed to test the interesting hypothesis that the vestibular system uses distinct channels of peripheral input to encode different features of natural vestibular stimuli.

\section{References}

Angelaki DE, Cullen KE (2008) Vestibular system: the many facets of a multimodal sense. Annu Rev Neurosci 31:125-150. CrossRef Medline

Angelaki DE, Dickman JD (2000) Spatiotemporal processing of linear accel- eration: primary afferent and central vestibular neuron responses. J Neurophysiol 84:2113-2132. Medline

Attneave F (1954) Some informational aspects of visual perception. Psychol Rev 61:183-193. CrossRef Medline

Baird RA, Desmadryl G, Fernández C, Goldberg JM (1988) The vestibular nerve of the chinchilla. II. Relation between afferent response properties and peripheral innervation patterns in the semicircular canals. J Neurophysiol 60:182-203. Medline

Barlow H (2001) Redundancy reduction revisited. Network 12:241-253. CrossRef Medline

Bell C, Maler L (2005) Central neuroanatomy of electrosensory systems in fish. In: Electroreception (Bullock TH, Hopkins CD, Popper AN, Fay RR, eds), pp 68-111. New York: Springer.

Brooks JX, Cullen KE (2013) The primate cerebellum selectively encodes unexpected self-motion. Curr Biol 23:947-955. CrossRef Medline

Brunel N, Nadal JP (1998) Mutual information, Fisher information, and population coding. Neural Comput 10:1731-1757. CrossRef Medline

Carr CE, Maler L (1986) Electroreception in gymnotiform fish: central anatomy and physiology. In: Electroreception (Bullock TH, Heiligenberg W, eds), pp 319-373. New York: Wiley.

Carriot J, Brooks JX, Cullen KE (2013) Multimodal integration of selfmotion cues in the vestibular system: active versus passive translations. J Neurosci 33:19555-19566. CrossRef Medline

Carriot J, Jamali M, Chacron MJ, Cullen KE (2014) Statistics of the vestibular input experienced during natural self-motion: implications for neural processing. J Neurosci 34:8347-8357. CrossRef Medline

Chichilnisky EJ (2001) A simple white noise analysis of neuronal light responses. Network 12:199-213. CrossRef Medline

Cover T, Thomas J (1991) Elements of Information Theory. New York: Wiley.

Cullen KE (2004) Sensory signals during active versus passive movement. Curr Opin Neurobiol 14:698-706. CrossRef Medline

Cullen KE (2011) Neural encoding of self-motion. Curr Opin Neurobiol 21:587-595. CrossRef Medline

Dale A, Cullen KE (2013) The nucleus prepositus predominantly outputs eye movement-related information during passive and active selfmotion. J Neurophysiol 109:1900-1911. CrossRef Medline

Domenici P, Blake R (1997) The kinematics and performance of fish faststart swimming. J Exp Biol 200:1165-1178. Medline

Domenici P, Standen EM, Levine RP (2004) Escape manoeuvres in the spiny dogfish (Squalus acanthias). J Exp Biol 207:2339-2349. CrossRef Medline

Eatock RA, Songer JE (2011) Vestibular hair cells and afferents: two channels for head motion signals. Annu Rev Neurosci 34:501-534. CrossRef Medline

Faisal AA, Selen LP, Wolpert DM (2008) Noise in the nervous system. Nat Rev Neurosci 9:292-303. CrossRef Medline

Fernández C, Goldberg JM (1976) Physiology of peripheral neurons innervating otolith organs of the squirrel monkey. II. Directional selectivity and force-response relations. J Neurophysiol 39:985-995. Medline

Fernández C, Baird RA, Goldberg JM (1988) The vestibular nerve of the chinchilla. I. Peripheral innervation patterns in the horizontal and superior semicircular canals. J Neurophysiol 60:167-181. Medline

Gelfand S (2004) Hearing: an introduction to psychological and physiological acoustics. Hoboken, NJ: Informa Healthcare.

Goldberg JM (2000) Afferent diversity and the organisation of central vestibular pathways. Exp Brain Res 130:277-297. CrossRef Medline

Goldberg JM, Fernández C (1977) Conduction times and background discharge of vestibular afferents. Brain Res 122:545-550. CrossRef Medline

Goldberg JM, Smith CE, Fernández C (1984) Relation between discharge regularity and responses to externally applied galvanic currents in vestibular nerve afferents of the squirrel monkey. J Neurophysiol 51:12361256. Medline

Hullar TE, Della Santina CC, Hirvonen T, Lasker DM, Carey JP, Minor LB (2005) Responses of irregularly discharging chinchilla semicircular canal vestibular-nerve afferents during high-frequency head rotations. J Neurophysiol 93:2777-2786. CrossRef Medline

Jamali M, Sadeghi SG, Cullen KE (2009) Response of vestibular nerve afferents innervating utricle and saccule during passive and active translations. J Neurophysiol 101:141-149. CrossRef Medline

Jamali M, Carriot J, Chacron MJ, Cullen KE (2013) Strong correlations between sensitivity and variability give rise to constant discrimination 
thresholds across the otolith afferent population. J Neurosci 33:1130211313. CrossRef Medline

Kalluri R, Xue J, Eatock RA (2010) Ion channels set spike timing regularity of mammalian vestibular afferent neurons. J Neurophysiol 104:20342051. CrossRef Medline

Kawasaki M (2005) Physiology of tuberous electrosensory systems. In: Electroreception (Bullock TH, Hopkins CD, Popper AN, Fay RR, eds), pp 154-194. New York: Springer.

Laughlin S (1981) A simple coding procedure enhances a neuron's information capacity. Z Naturforsch C 36:910-912. Medline

Lisberger SG, Pavelko TA (1986) Vestibular signals carried by pathways subserving plasticity of the vestibulo-ocular reflex in monkeys. J Neurosci 6:346-354. Medline

Livingstone MS, Hubel DH (1987) Psychophysical evidence for separate channels for the perception of form, color, movement, and depth. J Neurosci 7:3416-3468. Medline

Marr D (1982) Vision. New York: W.H. Freeman.

Massot C, Chacron MJ, Cullen KE (2011) Information transmission and detection thresholds in the vestibular nuclei: single neurons versus population encoding. J Neurophysiol 105:1798-1814. CrossRef Medline

Massot C, Schneider AD, Chacron MJ, Cullen KE (2012) The vestibular system implements a linear-nonlinear transformation in order to encode self-motion. PLoS Biol 10:e1001365. CrossRef Medline

McDonnell MD, Stocks NG (2008) Maximally informative stimuli and tuning curves for sigmoidal rate-coding neurons and populations. Phys Rev Lett 101:058103. CrossRef Medline

Merigan WH, Maunsell JH (1993) How parallel are the primate visual pathways? Annu Rev Neurosci 16:369-402. CrossRef Medline

Oertel D (1999) The role of timing in the brain stem auditory nuclei of vertebrates. Annu Rev Physiol 61:497-519. CrossRef Medline

Ramachandran R, Lisberger SG (2006) Transformation of vestibular signals into motor commands in the vestibuloocular reflex pathways of monkeys. J Neurophysiol 96:1061-1074. CrossRef Medline

Rieke F, Warland D, de Ruyter van Steveninck RR, Bialek W (1996) Spikes: exploring the neural code. Cambridge, MA: MIT.

Sadeghi SG, Minor LB, Cullen KE (2007a) Response of vestibular-nerve af- ferents to active and passive rotations under normal conditions and after unilateral labyrinthectomy. J Neurophysiol 97:1503-1514. CrossRef Medline

Sadeghi SG, Chacron MJ, Taylor MC, Cullen KE (2007b) Neural variability, detection thresholds, and information transmission in the vestibular system. J Neurosci 27:771-781. CrossRef Medline

Sadeghi SG, Goldberg JM, Minor LB, Cullen KE (2009) Effects of canal plugging on the vestibuloocular reflex and vestibular nerve discharge during passive and active head rotations. J Neurophysiol 102:2693-2703. CrossRef Medline

Si X, Zakir MM, Dickman JD (2003) Afferent innervation of the utricular macula in pigeons. J Neurophysiol 89:1660-1677. CrossRef Medline

Simoncelli EP, Olshausen BA (2001) Natural image statistics and neural representation. Annu Rev Neurosci 24:1193-1216. CrossRef Medline

Smith CE, Goldberg JM (1986) A stochastic afterhyperpolarization model of repetitive activity in vestibular afferents. Biol Cybern 54:41-51. CrossRef Medline

Stein RB, Gossen ER, Jones KE (2005) Neuronal variability: noise or part of the signal? Nat Rev Neurosci 6:389-397. CrossRef Medline

Steinacker A (2004) Sensory processing and ionic currents in vestibular hair cells. In: Springer Handbook of auditory research (Fay RR, Popper AN, eds), pp 202-234. New York: Springer.

Straka H, Vibert N, Vidal PP, Moore LE, Dutia MB (2005) Intrinsic membrane properties of vertebrate vestibular neurons: function, development and plasticity. Prog Neurobiol 76:349-392. CrossRef Medline

Takahashi T, Moiseff A, Konishi M (1984) Time and intensity cues are processed independently in the auditory system of the owl. J Neurosci 4:1781-1786. Medline

Wallman J, Velez J, Weinstein B, Green AE (1982) Avian vestibuloocular reflex-adaptive plasticity and developmental changes. J Neurophysiol 48 : 952-967. Medline

Wark B, Lundstrom BN, Fairhall A (2007) Sensory adaptation. Curr Opin Neurobiol 17:423-429. CrossRef Medline

Xue J, Peterson EH (2006) Hair bundle heights in the utricle: differences between macular locations and hair cell types. J Neurophysiol 95:171186. CrossRef Medline 